\title{
Dynamic stiffness of nonlocal damped nano-beams on elastic foundation
}

\author{
S. Adhikari ${ }^{\mathrm{a}}$, D. Karličića ${ }^{\mathrm{a}}$, X. Liu ${ }^{\mathrm{b}}$ \\ ${ }^{a}$ Future Manufacturing Research Institute, College of Engineering, Swansea University, Swansea, UK \\ ${ }^{b}$ Key Laboratory of Traffic Safety on Track, Ministry of Education, School of Traffic EG Transportation \\ Engineering, Central South University, Changsha, China
}

\begin{abstract}
Free and forced bending vibration of damped nonlocal nano-beams resting on an elastic foundation is investigated. Two types of nonlocal damping models, namely, strain-ratedependent viscous damping and velocity-dependent viscous damping are considered. A frequency-dependent dynamic finite element method is developed to obtain the forced vibration response. Frequency-adaptive complex-valued shape functions are used for the derivation of the dynamic stiffness matrix. It is shown that there are six unique coefficients which define the general dynamic stiffness matrix. These complex-valued coefficients are obtained exactly in closed-form and illustrated numerically as functions of the frequency. It is proved that the general dynamic stiffness matrix reduces to the well known special cases under different conditions. The stiffness and mass matrices of the nonlocal beam are also obtained using the conventional finite element method. A numerical algorithm to extract the eigenvalues from the dynamic stiffness matrix with a transcendental element for the special case when the system is undamped is suggested. Results from the dynamic finite element method and the conventional finite element method are compared. The application of the dynamic stiffness approach is shown through forced response analysis of a double-walled carbon nanotube in pinned-pinned and cantilever configurations. Explicit closed-form expressions of the dynamic response for both the cases have been obtained and the role of crucial system parameters such as, the damping factors, the nonlocal parameter and the foundation stiffness have been investigated.
\end{abstract}

Keywords: bending vibration; nonlocal mechanics; dynamic stiffness; asymptotic analysis; frequency response

\section{Introduction}

The dynamics of damped structures is of great significance in many engineering applications on macro as well as on micro- and nano-scales [1]. For this reason, studying the steady-state, and transient motion in nanostructure systems has been an exciting perspective in the last few years [2]. Here, we will analyse the free and forced vibration of damped

${ }^{*}$ Corresponding author. Tel: +44 (0)1792 602088, Fax: +44 (0)1792 295676

Email addresses: S.Adhikari@swansea.ac.uk (S. Adhikari), danilo.karlicic@swansea.ac.uk (D. Karličić), xiangliu@csu.edu.cn (X. Liu) 
beam-like nanostructures placed on the elastic foundation by using the finite element discretisation and dynamic stiffness matrix. Investigation of the frequency response function (FRF) is extremely important in order to determine the responses of a given system and their application to real nanoengineering structures. The various interesting problems can occur in micro-electromechanical systems (MEMS), nano-electromechanical systems (NEMS) devices, where the structural collapse can be caused by very small external load at the resonant frequency. Theoretical analysis of nano-scale systems become an important task in design procedures and development of new types of micro- and nano-devices [3]. Special classes of beam-like nanostructures are related to single and multi-walled carbon nanotube [4], boron nitride nanotubes (BNNTs) [5] and Zinc Oxide nanotubes [6]. There are many examples of mathematical models studying the vibration behaviour of beam-like nanostructures in piezoelectric sensors and actuators, MEMS, NEMS [7] and atomic force microscopes [8]. Special attention of the scientific community is related to the vibration analyses of beam nanostructures, which become an important topic especially in the design of NEMS devices.

By conducting experiments, it is found that small-scale effects play a significant role in the physical behaviour of nanostructures. However, performing experiments on the nanoscale level is not an easy task due to weak control of parameters in the experiment and considerable cost. Therefore, many investigators focused on theoretical methods splitting in two different directions. The first method is based on atomistic models accounting discrete nature of nanostructures such as molecular dynamics, density function theory etc. These methods are shown to be efficient only for the systems with a small number of particles and time-consuming for more complex nanostructure systems [9]. Another approach is based on modified continuum theories accounting size effects, which allows us to use standard methods from structural mechanics to model and solve different dynamic and stability problems of simpler and complex nanostructure systems. One among the first such theories that account size effects through a single material parameter was the nonlocal theory of elasticity established by Eringen [10,11]. This theory is shown to be very effective in describing the mechanical behaviour of various nanostructures and nanocomposites compared to other methods. It is important to note that the value of the nonlocal parameter is usually obtained by fitting the results from the nonlocal continuum model with the molecular dynamics simulation results for natural frequencies or dispersion curves for Born-Karman model of lattice dynamics.

One of the first studies of bending of a nanobeam using nonlocal continuum mechanics for different boundary conditions was proposed by Peddinson et al. [12]. They investigated the influence of the material length-scale parameter on the deflection of a Euler-Bernoulli nanobeam model and concluded that the nonlocal effect is important in the development of a micro/nano-scale device. Murmu and Adhikari [13, 14] developed a nonlocal double-nanobeam model to investigate the natural frequency for simply supported boundary conditions. They found that the small-scale effects in the vibrating system are higher with increasing values of the nonlocal parameter for the case of in-phase modes of vibration than in the out-of-phase modes of vibration. Reddy [15] reformulated the local nanobeam theory by using nonlocal differential constitutive relations of Eringen to analytically study bending, vibration and buckling behaviours of a nanobeam. Reddy and Pang [16] derived an analytical solution of bending, vibration and buckling based on the Eringen nonlocal elasticity theory for a carbon nanotube. Eltaher et al. 
[17] considered free vibrations of a Euler-Bernoulli nanobeam by using the finite element method. They showed the effects of nonlocal parameter, slenderness ratios, rotator inertia and boundary conditions on the fundamental frequency of the nanobeam. Phadikar and Pradhan [18] derived a variation formulation and the Finite Element (FE) analysis for the nonlocal nanobeams and nanoplates models. They obtained corresponding numerical framework for analysing the bending, vibration and buckling of the nonlocal beam with four classical boundary conditions. Based on the Euler-Bernoulli beam theory, integral nonlocal elasticity and FE analysis, Tuna and Kirca [19] analysed free vibration behaviour of nanobeam structures. Pradhan [20] investigated the FE model for bending, buckling and vibration of nonlocal beams with clamped-clamped, hinged-hinged, clamped-hinged and clamped-free boundary conditions, based on the nonlocal elasticity theory. Adhikari et al. [21-23] analysed free and forced vibration of an axial rod vibration based on the dynamic stiffness matrix method. They show an advance methodology for the investigation of the frequency response of the nonlocal nanostructures.

Based on the above discussion, in this paper, we develop the dynamic finite element based on nonlocal elasticity with the aim of considering modal analysis and dynamic response analysis of beams undergoing bending vibrations. The dynamic finite element method belongs to the general class of spectral methods for linear dynamical systems [24]. This approach, or approaches very similar to this, is known by various names such as the dynamic stiffness method [25-31], spectral finite element method [24, 32] and dynamic finite element method [33, 34]. So far the dynamic finite element method has been applied to classical local beams only. In this paper, we generalise this approach to nonlocal beam resting on an elastic foundation. This is useful, for example, dynamic response analysis of nanotubes and nanowires resting on a subgrade. One of the novel features of the analysis proposed here is the employment of frequency-dependent complex nonlocal shape functions for damped systems. This, in turn, enables us to obtain the element stiffness matrix using the usual weak form of the finite element method.

Overview of the paper is as follows. In Section 2 we briefly introduce the theory of nonlocal elasticity and describe the equation of motion for bending vibration of undamped and damped beams resting on elastic foundation. The conventional finite element method and the dynamic finite element method are developed in Section 3. Closed-form expressions are derived for the conventional mass and stiffness matrices and the complex-valued dynamic stiffness matrix. We explicitly demonstrate how the general dynamic stiffness matrix reduces to well known special cases under different conditions. Free vibration analysis of nonlocal beams is discussed in Section 4 using the Wittrick-Williams algorithm. In Section 5 the proposed methodology is applied to an armchair double-walled carbon nanotube (DWCNT) for illustration. The evolution of the dynamic stiffness coefficients as functions of the frequency is numerically illustrated. The dynamic response obtained from the conventional finite element method is compared with the dynamic stiffness approach. Finally, in Section 6 some conclusions are drawn based on the results obtained in the paper. 


\section{Bending vibration of undamped and damped nonlocal beams on elastic foundation}

\subsection{Introduction to nonlocal elasticity}

A well-known assumption of the nonlocal elasticity theory is that the stress at a point $x$ within an elastic body is a function not only of the strain at that point $x$, but is also a function of strains at all the other points within that body. Eringen and co-workers $[10,11]$ introduced the nonlocal elasticity constitutive equation with a single material parameter that takes into account small-scale effects and discrete nature of materials. The integral form [11] of a nonlocal linear constitutive relation for a three-dimensional body is given as

$$
\begin{gathered}
\sigma_{i j}(x)=\int_{V} \alpha\left(\left|x-x^{\prime}\right|, \tau\right) t_{i j}\left(x^{\prime}\right) d V\left(x^{\prime}\right), \quad \forall x \in V \\
\sigma_{i j, i}=\rho\left(\ddot{u}_{j}-f_{j}\right) \\
\varepsilon_{i j}\left(x^{\prime}\right)=\frac{1}{2}\left(u_{i, j}\left(x^{\prime}\right)+u_{j, i}\left(x^{\prime}\right)\right) \\
t_{i j}\left(x^{\prime}\right)=\lambda \varepsilon_{r r}\left(x^{\prime}\right) \delta_{i j}+2 \mu \varepsilon_{i j}\left(x^{\prime}\right)
\end{gathered}
$$

Here $\sigma_{i j}$ and $\varepsilon_{i j}$ are respectively the stress and strain tensors, $u_{j}$ is the displacement vector and $f_{j}$ is the body force, $t_{i j}\left(x^{\prime}\right)$ is the macroscopic (classical) stress tensor at $x^{\prime}$ which is related to the linear strain tensor $\varepsilon_{i j}\left(x^{\prime}\right)$ at any point $x^{\prime}$ in the body, where $\lambda$ and $\mu$ represents Lame's constants. In Eq. (1), $\alpha\left(\left|x-x^{\prime}\right|, \tau\right)$ denotes the nonlocal modulus or attenuation function that incorporates nonlocal effects into the constitutive equation at a reference point $x$ produced by the local strain at a source $x^{\prime}$. The above absolute value of the difference $\left|x-x^{\prime}\right|$ denotes the Euclidean metric. In the parameter $\tau=\left(e_{0} a\right) / l$, the characteristic length (crack length, wavelength) is denoted with $l$. This can describe an internal characteristic length such as, a lattice parameter, granular size and the distance between $\mathrm{C}-\mathrm{C}$ bonds. The parameter $e_{0}$ is a constant appropriate to each material that can be identified from atomistic simulations or by using the dispersive curve of the Born-Karman model of lattice dynamics.

Since it is not an easy task to solve particular governing equations when the integral form of nonlocal constitutive relation is employed, one should use a differential form of nonlocal elasticity constitutive relation for the one-dimensional case as

$$
\left[1-\left(e_{0} a\right)^{2} \frac{\partial^{2}}{\partial x^{2}}\right] \sigma_{x x}=E \varepsilon_{x x}
$$

where $\left(e_{0} a\right)^{2}$ is the nonlocal parameter (length scales), $E$ is the Young elastic modulus. Since the exact value of the nonlocal parameter is scattered and depends on applied boundary conditions, material and geometric properties of nanostructures, in the present study we assumed the value of $e_{0} a$ to be in the range $0-2 \mathrm{~nm}$. When $e_{0} a=0$, there is no influence of nonlocal parameter (same as in macro-scale modelling) and we get back to classical stress-strain relation of an elastic body. By combining the constitutive relation given in (5) along with the assumptions form the Euler-Bernoulli beam theory, the governing equation of motion can be derived, as it showed in the next section. 


\subsection{Undamped system}

For the bending vibration of a nonlocal damped beam resting on a nonlocal elastic foundation, the equation of motion [35-37] can be expressed by

$$
\begin{aligned}
E I \frac{\partial^{4} V(x, t)}{\partial x^{4}}+\left(1-\left(e_{0} a\right)^{2} \frac{\partial^{2}}{\partial x^{2}}\right)\left\{m \frac{\partial^{2} V(x, t)}{\partial t^{2}}+k V(x, t)\right\} & \\
& =\left(1-\left(e_{0} a\right)^{2} \frac{\partial^{2}}{\partial x^{2}}\right)\{F(x, t)\}
\end{aligned}
$$

An illustrative diagram of the system is shown in Fig. 1 In the above equation, EI is

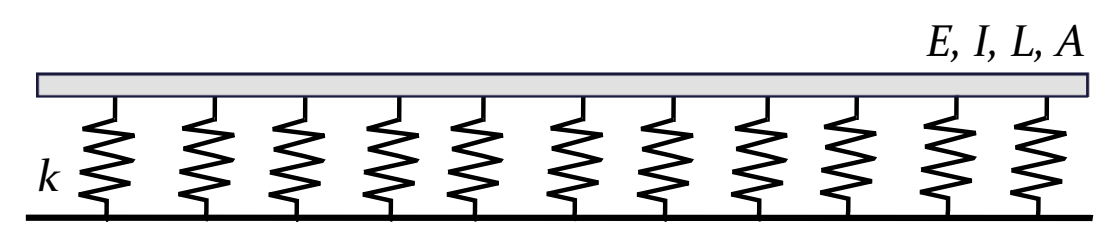

Fig. 1: A nonlocal Euler-Bernoulli beam on elastic foundation. Here $A$ is the cross sectional area, $E$ is the Young's modulus of the material, $I$ is area the moment of inertia, $m$ is mass per unit length, $k$ is the stiffness per unit length of the elastic foundation and $L$ is the length of the beam. Different boundary conditions can be applied at the both ends of the beam.

the bending rigidity, $m$ is mass per unit length, $k$ is the stiffness per unit length of the elastic foundation, $e_{0} a$ is the nonlocal parameter, $V(x, t)$ is the transverse displacement and $F(x, t)$ is the applied force. In some cases, the value of the nonlocal parameter can be obtained from fundamental principles [38, 39]. It may be noted that the nonlocal parameter value can be dependent on the physics of the problem (e.g., buckling, vibration) as well as on the boundary conditions (e.g., fixed, free, pinned). In literature, both differential and integral forms of the nonlocal model have been employed [40, 41]. Here we employ the differential form. Considering the free vibration, i.e., setting the force to zero, and assuming harmonic motion with frequency $\omega$

$$
V(x, t)=v(x) \exp [\mathrm{i} \omega t]
$$

Substituting this in Eq. (6) we have

$$
\begin{gathered}
E I \frac{d^{4} v}{d x^{4}}-\left(m \omega^{2}-k\right)\left(v-\left(e_{0} a\right)^{2} \frac{d^{2} v}{d x^{2}}\right)=0 \\
\text { or } \quad \frac{d^{4} v}{d x^{4}}+b^{4}\left(e_{0} a\right)^{2} \frac{d^{2} v}{d x^{2}}-b^{4} v=0
\end{gathered}
$$

In the above equation, for any positive value of frequency $\omega$, the frequency dependent constant $b$ can be written as

$$
b^{4}=\frac{m \omega^{2}-k}{E I}=\frac{m \omega^{2}}{E I}\left(1-\frac{k}{m \omega^{2}}\right)=c^{4}\left(1-\omega_{s}^{2} / \omega^{2}\right)
$$

Here the frequency dependent constant $c$ and the elastic medium natural frequency $\omega_{s}$ are defined as

$$
c^{4}=\frac{m \omega^{2}}{E I} \quad \text { and } \quad \omega_{s}^{2}=\frac{k}{m}
$$

To obtain the characteristic equation we assume

$$
v(x)=\exp [\lambda x]
$$


Substituting this in Eq. (9) we obtain

$$
\begin{array}{ll} 
& \lambda^{4}+b^{4}\left(e_{0} a\right)^{2} \lambda^{2}-b^{4}=0 \\
\text { or } \quad & \lambda^{2}=b^{2}\left(-b^{2}\left(e_{0} a\right)^{2} \pm \sqrt{4+b^{4}\left(e_{0} a\right)^{4}}\right) / 2
\end{array}
$$

Defining

$$
\gamma=b^{2}\left(e_{0} a\right)^{2}
$$

the two roots can be expressed as

$$
\lambda^{2}=-\alpha^{2}, \beta^{2}
$$

where

$$
\begin{aligned}
\alpha & =b \sqrt{\left(\sqrt{4+\gamma^{2}}+\gamma\right) / 2} \\
\text { and } \beta & =b \sqrt{\left(\sqrt{4+\gamma^{2}}-\gamma\right) / 2}
\end{aligned}
$$

Therefore, the four roots of the characteristic equation can be expressed as

$$
\lambda=\mathrm{i} \alpha,-\mathrm{i} \alpha, \beta,-\beta
$$

where $\mathrm{i}=\sqrt{-1}$ is the unit imaginary number. The displacement function within the beam can be expressed by linear superposition as

$$
v(x)=\sum_{j=1}^{4} c_{j} \exp \left[\lambda_{j} x\right]
$$

Here the unknown constants $c_{j}$ need to be obtained from the boundary conditions.

Using Eq. (20), the natural frequency of the system are obtained by imposing the necessary boundary conditions [42]. For example, the bending moment and shear force are given by:

- Bending moment at $x=0$ or $x=L$ :

$$
E I \frac{d^{2} v(x)}{d x^{2}}-m \omega^{2}\left(e_{0} a\right)^{2} v(x)=0 \quad \text { or } \quad \frac{d^{2} v(x)}{d x^{2}}-c^{4}\left(e_{0} a\right)^{2} v(x)=0
$$

- Shear force at $x=0$ or $x=L$ :

$$
E I \frac{d^{3} v(x)}{d x^{3}}+\left(m \omega^{2}-k\right)\left(e_{0} a\right)^{2} \frac{d v(x)}{d x}=0 \quad \text { or } \quad \frac{d^{3} v(x)}{d x^{3}}+b^{4}\left(e_{0} a\right)^{2} \frac{d v(x)}{d x}=0
$$

Boundary conditions involving displacements and rotations can be applied in a conventional manner. There are different ways in which the nonlocal boundary condition can be applied. Several authors have shown inconsistent results in the application of the Eringen differential constitutive relation in modelling the cantilever beam by comparing with other types of boundary conditions [43, 44]. Moreover, it is stated that for all boundary conditions except the cantilever, application of the differential form of constitutive relation in modelling nano-beam predicts softening effect for increasing of the nonlocal parameter as is stated in [44]. In order to overcome this paradox, which appear in the boundary conditions, there are few approaches. One of the first paper is proposed by 
Challamel and Wang [45], showed that the gradient elastic model as well as an integral nonlocal elastic model that is based on combining the local and the non-local curvatures in the constitutive elastic relation. Then, in [46], the authors presented a two-phase unified integro-differential nonlocal model for analysing the cantilever Euler-Bernoulli beam where the finite element method is employed in order to solve the bending problem, where the problem of the cantilever beam is also included. On the other hand, in [47, 48], the authors proposed the application of a stress-driven integral constitutive law instead of the Eringen strain-driven integral constitutive law, in order to get well-posed nonlocal elastic problems for applications to structural mechanics. We refer to the papers by Challamel et. al $[49,50]$ for further comprehensive discussions. The focus of this paper is the derivation of the element dynamic stiffness matrix of the beam. This matrix captures the relationship between applied forces and displacements at the two nodes of the beam. As a result, the boundary conditions do not impact the derivation of the element dynamic stiffness matrix. The resulting dynamic response, of course, will depend on the applied boundary conditions as will be seen later in the paper.

\subsection{Damped system}

The equation of motion of bending vibration for a damped nonlocal beam can be expressed as

$$
\begin{aligned}
E I \frac{\partial^{4} V(x, t)}{\partial x^{4}}+(1- & \left.\left(e_{0} a\right)^{2} \frac{\partial^{2}}{\partial x^{2}}\right)\left\{m \frac{\partial^{2} V(x, t)}{\partial t^{2}}+k V(x, t)\right\}+\widehat{c}_{1}\left(1-\left(e_{0} a\right)_{1}^{2} \frac{\partial^{2}}{\partial x^{2}}\right) \frac{\partial^{5} V(x, t)}{\partial x^{4} \partial t} \\
& +\widehat{c}_{2}\left(1-\left(e_{0} a\right)_{2}^{2} \frac{\partial^{2}}{\partial x^{2}}\right) \frac{\partial V(x, t)}{\partial t}=\left(1-\left(e_{0} a\right)^{2} \frac{\partial^{2}}{\partial x^{2}}\right)\{F(x, t)\} \quad(23)
\end{aligned}
$$

Here $\widehat{c}_{1}$ is the strain-rate-dependent viscous damping coefficient and $\widehat{c}_{2}$ is the velocitydependent viscous damping coefficient. The parameters $\left(e_{0} a\right)_{1}$ and $\left(e_{0} a\right)_{2}$ are nonlocal parameters related to the two damping terms respectively. For simplicity, we have not taken into account any nonlocal effect related to the damping. Although this can be mathematically incorporated in the analysis, the determination of these nonlocal parameters is beyond the scope of this work and therefore only local interaction for the damping is adopted. Thus, in the following analysis, we consider $\left(e_{0} a\right)_{1}=\left(e_{0} a\right)_{2}=0$.

Assuming harmonic response as in Eq. (7) and considering free vibration, from Eq. (23) we have

$$
E I \frac{d^{4} v}{d x^{4}}-\left(m \omega^{2}-k\right)\left(v-\left(e_{0} a\right)^{2} \frac{d^{2} v}{d x^{2}}\right)+\mathrm{i} \omega \widehat{c}_{1} \frac{d^{4} v}{d x^{4}}+\mathrm{i} \omega \widehat{c}_{2} v=0
$$

Following the damping convention in dynamic analysis [42], we consider stiffness and mass proportional damping. Therefore, we express the damping constants as

$$
\widehat{c}_{1}=\zeta_{1} E I \text { and } \widehat{c}_{2}=\zeta_{2} m
$$

where $\zeta_{1}$ and $\zeta_{2}$ are stiffness and mass proportional damping factors. Substituting these, from Eq. (24) we have

$$
\begin{aligned}
& E I\left(1+\mathrm{i} \omega \zeta_{1}\right) \frac{d^{4} v}{d x^{4}}+\left(m \omega^{2}-k\right)\left(e_{0} a\right)^{2} \frac{d^{2} v}{d x^{2}}-\left(m \omega^{2}-k\right)\left(1-\mathrm{i} \zeta_{2} / \omega\right) v=0 \\
& \text { or } \quad \frac{d^{4} v}{d x^{4}}+\bar{b}^{4}\left(e_{0} a\right)^{2} \frac{d^{2} v}{d x^{2}}-\bar{b}^{4} \theta v=0
\end{aligned}
$$


where we define $\bar{b}$ and introduce $\theta$ as

$$
\bar{b}^{4}=\frac{\left(m \omega^{2}-k\right)}{E I\left(1+\mathrm{i} \omega \zeta_{1}\right)} \quad \text { and } \quad \theta=\left(1-\mathrm{i} \zeta_{2} / \omega\right)
$$

Comparing this with the equivalent expression for the undamped case in Eq. (10) we notice that $\bar{b}^{4}$ is a complex function of the frequency parameter $\omega$ as opposed to a real function. In the special case when damping coefficients $\zeta_{1}$ and $\zeta_{1}$ go to zero, $\bar{b}^{4}$ in Eq. (28) reduces to the expression of $b^{4}$ in Eq. (10) and $\theta$ becomes 1 . The characteristics equation can be obtained in manned similar to the undamped system with the expressions of $\alpha$ and $\beta$ being

$$
\begin{aligned}
\alpha & =\bar{b} \sqrt{\left(\sqrt{4 \theta+\gamma^{2}}+\gamma\right) / 2} \\
\text { and } \beta & =\bar{b} \sqrt{\left(\sqrt{4 \theta+\gamma^{2}}-\gamma\right) / 2}
\end{aligned}
$$

Here $\gamma=\bar{b}^{2}\left(e_{0} a\right)^{2}$ is now a complex valued function of the frequency.

\section{The dynamic finite element matrix}

\subsection{Classical finite element of nonlocal beams}

We first consider standard finite element analysis of the nonlocal beam. Phadikar and Pradhan [51] proposed a variational-formulation-based finite element approach for nanobeams and nanoplates. We consider an element of length $L$ with bending stiffness $E I$, elastic foundation stiffness $k$ and mass per unit length $m$. An element of the beam is shown in Fig. 2. This element has four degrees of freedom and there are four shape

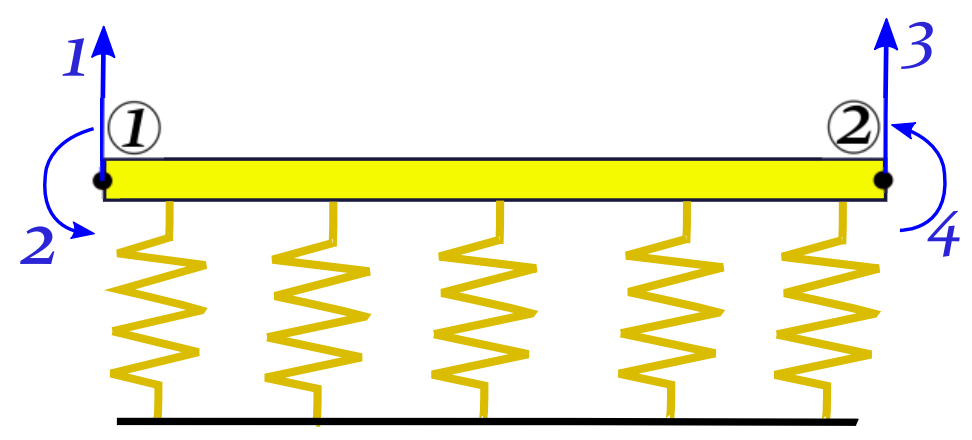

Fig. 2: A nonlocal element for the bending vibration of a beam. It has two nodes and four degrees of freedom. The displacement field within the element is expressed by cubic shape functions for the classical finite element analysis. Complex valued frequency-dependent transcendental shape functions are used for the proposed dynamic finite element analysis.

functions. The shape function matrix for the bending deformation [52] can be given by

$$
\mathbf{N}(x)=\left[N_{1}(x), N_{2}(x), N_{3}(x), N_{4}(x)\right]^{T}
$$

where

$$
\begin{array}{ll}
N_{1}(x)=1-3 \frac{x^{2}}{L^{2}}+2 \frac{x^{3}}{L^{3}}, & N_{2}(x)=x-2 \frac{x^{2}}{L}+\frac{x^{3}}{L^{2}}, \\
N_{3}(x)=3 \frac{x^{2}}{L^{2}}-2 \frac{x^{3}}{L^{3}}, & N_{4}(x)=-\frac{x^{2}}{L}+\frac{x^{3}}{L^{2}}
\end{array}
$$


Using this, the stiffness matrix can be obtained following the conventional variational formulation [53] as

$$
\begin{aligned}
& \mathbf{K}_{e}=E I \int_{0}^{L} \frac{d^{2} \mathbf{N}(x)}{d x^{2}} \frac{d^{2} \mathbf{N}^{T}(x)}{d x^{2}} \mathrm{~d} x+k \int_{0}^{L} \mathbf{N}(x) \mathbf{N}^{T}(x) \mathrm{d} x+k\left(e_{0} a\right)^{2} \int_{0}^{L} \frac{d \mathbf{N}(x)}{d x} \frac{d \mathbf{N}^{T}(x)}{d x} \mathrm{~d} x \\
& =\frac{E I}{L^{3}}\left[\begin{array}{cccc}
12 & 6 L & -12 & 6 L \\
6 L & 4 L^{2} & -6 L & 2 L^{2} \\
-12 & -6 L & 12 & -6 L^{2} \\
6 L & 2 L^{2} & -6 L & 4 L^{2}
\end{array}\right]+\frac{k L}{420}\left[\begin{array}{cccc}
156 & 22 L & 54 & -13 L \\
22 L & 4 L^{2} & 13 L & -3 L^{2} \\
54 & 13 L & 156 & -22 L \\
-13 L & -3 L^{2} & -22 L & 4 L^{2}
\end{array}\right] \\
& +\left(\frac{e_{0} a}{L}\right)^{2} \frac{k L}{30}\left[\begin{array}{cccc}
36 & 3 L & -36 & 3 L \\
3 L & 4 L^{2} & -3 L & -L^{2} \\
-36 & -3 L & 36 & -3 L \\
3 L & -L^{2} & -3 L & 4 L^{2}
\end{array}\right]
\end{aligned}
$$

The mass matrix for the nonlocal element can be obtained as

$$
\begin{aligned}
\mathbf{M}_{e} & =m \int_{0}^{L} \mathbf{N}(x) \mathbf{N}^{T}(x) \mathrm{d} x+m\left(e_{0} a\right)^{2} \int_{0}^{L} \frac{d \mathbf{N}(x)}{d x} \frac{d \mathbf{N}^{T}(x)}{d x} \mathrm{~d} x \\
& =\frac{m L}{420}\left[\begin{array}{cccc}
156 & 22 L & 54 & -13 L \\
22 L & 4 L^{2} & 13 L & -3 L^{2} \\
54 & 13 L & 156 & -22 L \\
-13 L & -3 L^{2} & -22 L & 4 L^{2}
\end{array}\right]+\left(\frac{e_{0} a}{L}\right)^{2} \frac{m L}{30}\left[\begin{array}{cccc}
36 & 3 L & -36 & 3 L \\
3 L & 4 L^{2} & -3 L & -L^{2} \\
-36 & -3 L & 36 & -3 L \\
3 L & -L^{2} & -3 L & 4 L^{2}
\end{array}\right]
\end{aligned}
$$

For the special case when the beam is local, the mass matrix derived above reduces to the classical mass matrix $[52,53]$ as $e_{0} a=0$. It should be noted that since the shape function in the classical finite element method is not exact for dynamic problems, a number of elements should be used to model a beam member. Therefore, the elemental stiffness $\mathbf{K}_{e}$ and mass $\mathbf{M}_{e}$ matrices should be assembled to form global stiffness $\mathbf{K}_{g}$ and mass $\mathbf{M}_{g}$ matrices and finally global dynamic stiffness matrix of the overall structure based the above classical finite element method becomes

$$
\mathbf{D}_{g}(\omega)=\mathbf{K}_{g}-\omega^{2} \mathbf{M}_{g}
$$

and the equation of dynamic equilibrium is

$$
\mathrm{D}_{g}(\omega) \widehat{\mathbf{v}}_{g}(\omega)=\widehat{\mathbf{f}}_{g}(\omega)
$$

which can be used for forced vibration directly. For free vibration analysis, natural frequencies $\omega$ are computed for when $\left(\mathbf{K}_{g}-\omega^{2} \mathbf{M}_{g}\right) \widehat{\mathbf{v}}_{g}(\omega)=\mathbf{0}$ has nontrivial solutions $\widehat{\mathbf{v}}_{g}$ and will be mentioned later in Section 4 .

\subsection{Dynamic finite element for damped nonlocal beam}

The first step for the derivation of the dynamic element matrix is the generation of dynamic shape functions. The dynamic shape functions are obtained such that the equation of dynamic equilibrium is satisfied exactly at all points within the element. Similarly to the classical finite element method, assume that the frequency-dependent displacement within an element is interpolated from the nodal displacements as

$$
v_{e}(x, \omega)=\mathbf{N}^{T}(x, \omega) \widehat{\mathbf{v}}_{e}(\omega)
$$


Here $\widehat{\mathbf{v}}_{e}(\omega) \in \mathbb{C}^{n}$ is the nodal displacement vector $\mathbf{N}(x, \omega) \in \mathbb{C}^{n}$ is the vector of frequencydependent shape functions and $n=4$ is the number of the nodal degrees-of-freedom. Suppose the $s_{j}(x, \omega) \in \mathbb{C}, j=1, \cdots, 4$ are the basis functions which exactly satisfy Eq. (27). It can be shown that the shape function vector can be expressed as

$$
\mathbf{N}(x, \omega)=\mathbf{\Gamma}(\omega) \mathbf{s}(x, \omega)
$$

where the vector $\mathbf{s}(x, \omega)=\left\{s_{j}(x, \omega)\right\}^{T}, \forall j=1, \cdots, 4$ and the complex matrix $\boldsymbol{\Gamma}(\omega) \in$ $\mathbb{C}^{4 \times 4}$ depends on the boundary conditions. The elements of $\mathbf{s}(x, \omega)$ constitutes $\exp \left[\lambda_{j} x\right]$ where the values of $\lambda_{j}$ are obtained from the solution of the characteristics equation as given in Eq. (19). An element for the damped beam under bending vibration is shown in Fig. 2. The degrees-of-freedom for each nodal point include a vertical and a rotational degrees-of-freedom.

In view of the solutions in Eq. (19), the displacement field with the element can be expressed by linear combination of the basic functions $e^{-\mathrm{i} \alpha x}, e^{\mathrm{i} \alpha x}, e^{\beta x}$ and $e^{-\beta x}$ so that in our notations $\mathbf{s}(x, \omega)=\left\{e^{-\mathrm{i} \alpha x}, e^{\mathrm{i} \alpha x}, e^{\beta x}, e^{-\beta x}\right\}^{T}$. We can also express $\mathbf{s}(x, \omega)$ in terms of trigonometric functions. Considering $e^{ \pm \mathrm{i} \alpha x}=\cos (\alpha x) \pm \mathrm{i} \sin (\alpha x)$ and $e^{ \pm \beta x}=$ $\cosh (\beta x) \pm \mathrm{i} \sinh (\beta x)$, the vector $\mathbf{s}(x, \omega)$ can be alternatively expressed as

$$
\mathbf{s}(x, \omega)=\left\{\begin{array}{c}
\sin (\alpha x) \\
\cos (\alpha x) \\
\sinh (\beta x) \\
\cosh (\beta x)
\end{array}\right\} \in \mathbb{C}^{4}
$$

The displacement field within the element can be expressed as

$$
v(x)=\mathbf{s}(x, \omega)^{T} \mathbf{v}_{e}
$$

where $\mathbf{v}_{e} \in \mathbb{C}^{4}$ is the vector of constants to be determined from the boundary conditions.

The relationship between the shape functions and the boundary conditions can be represented as in Table 1, where boundary conditions in each column give rise to the corresponding shape function. Writing Eq. (40) for the above four sets of boundary

\begin{tabular}{c|cccc} 
& $N_{1}(x, \omega)$ & $N_{2}(x, \omega)$ & $N_{3}(x, \omega)$ & $N_{4}(x, \omega)$ \\
\hline$v(0)$ & 1 & 0 & 0 & 0 \\
$\frac{\mathrm{d} v}{\mathrm{~d} x}(0)$ & 0 & 1 & 0 & 0 \\
$v(L)$ & 0 & 0 & 1 & 0 \\
$\frac{\mathrm{d} v}{\mathrm{~d} x}(L)$ & 0 & 0 & 0 & 1
\end{tabular}

Table 1: The relationship between the boundary conditions and the shape functions for the bending vibration of beams.

conditions, one obtains

$$
[\mathbf{R}]\left[\mathbf{v}_{e}^{1}, \mathbf{v}_{e}^{2}, \mathbf{v}_{e}^{3}, \mathbf{v}_{e}^{4}\right]=\mathbf{I}
$$

where

$$
\mathbf{R}=\left[\begin{array}{cccc}
s_{1}(0) & s_{2}(0) & s_{3}(0) & s_{4}(0) \\
\frac{\mathrm{d} s_{1}}{\mathrm{~d} x}(0) & \frac{\mathrm{d} s_{2}}{\mathrm{~d} x}(0) & \frac{\mathrm{d} s_{3}}{\mathrm{~d} x}(0) & \frac{\mathrm{d} s_{4}}{\mathrm{~d} x}(0) \\
s_{1}(L) & s_{2}(L) & s_{3}(L) & s_{4}(L) \\
\frac{\mathrm{d} s_{1}}{\mathrm{~d} x}(L) & \frac{\mathrm{d} s_{2}}{\mathrm{~d} x}(L) & \frac{\mathrm{d} s_{3}}{\mathrm{~d} x}(L) & \frac{\mathrm{d} s_{4}(L)}{\mathrm{d} x}(L)
\end{array}\right]
$$


and $\mathbf{v}_{e}^{k}$ is the vector of constants giving rise to the $k$ th shape function. In view of the boundary conditions represented in Table 1 and equation (41), the shape functions for bending vibration can be shown to be given by Eq. (38) where

$$
\boldsymbol{\Gamma}(\omega)=\left[\mathbf{v}_{e}^{1}, \mathbf{v}_{e}^{2}, \mathbf{v}_{e}^{3}, \mathbf{v}_{e}^{4}\right]^{T}=\left[\mathbf{R}^{-1}\right]^{T}
$$

By obtaining the matrix $\Gamma(\omega)$ from the above equation, the shape function vector can be obtained from Eq. (38).

The stiffness and mass matrices can be obtained similarly to the static finite element case discussed before. Note that for this case all the matrices become complex and frequency-dependent, which is different from the finite element matrices of Eq. (35). The dynamic stiffness matrix (omitting the subscript) is therefore

$$
\mathbf{D}(\omega)=\mathbf{K}_{e}(\omega)-\omega^{2} \mathbf{M}_{e}(\omega)
$$

and dynamic equilibrium can be also formulated as

$$
\mathbf{D}_{e}(\omega) \widehat{\mathbf{v}}_{e}(\omega)=\widehat{\mathbf{f}}(\omega)
$$

In Eq. (44), the frequency-dependent stiffness and mass matrices can be obtained as

$$
\begin{aligned}
\mathbf{K}_{e}(\omega) & =E I \int_{0}^{L} \frac{d^{2} \mathbf{N}(x, \omega)}{d x^{2}} \frac{d^{2} \mathbf{N}^{T}(x, \omega)}{d x^{2}} \mathrm{~d} x \\
\text { and } \mathbf{M}_{e}(\omega) & =m \int_{0}^{L} \mathbf{N}(x, \omega) \mathbf{N}^{T}(x, \omega) \mathrm{d} x
\end{aligned}
$$

After some algebraic simplifications similar to [27, 54], it can be shown that the dynamic stiffness matrix is given by the following closed-form expression

$$
\mathbf{D}(\omega)=E I\left[\begin{array}{cccc}
d_{1} & d_{2} & d_{4} & d_{5} \\
& d_{3} & -d_{5} & d_{6} \\
& & d_{1} & -d_{2} \\
\text { sym } & & & d_{3}
\end{array}\right]
$$

where the matrix coefficients are

$$
\begin{aligned}
d_{1} & =-\alpha \beta\left(\alpha^{2}+\beta^{2}\right)(C \alpha s+c \beta S) / \Delta \\
d_{2} & =-\alpha \beta\left(2 s S \alpha \beta+\left(\beta^{2}-\alpha^{2}\right)(C c-1)\right) / \Delta \\
d_{3} & =-(C \beta s-\alpha c S)\left(\alpha^{2}+\beta^{2}\right) / \Delta \\
d_{4} & =\alpha \beta\left(\alpha^{2}+\beta^{2}\right)(\beta S+\alpha s) / \Delta \\
d_{5} & =-\alpha \beta(C-c)\left(\alpha^{2}+\beta^{2}\right) / \Delta \\
\text { and } \quad d_{6} & =\left(\alpha^{2}+\beta^{2}\right)(-\alpha S+\beta s) / \Delta
\end{aligned}
$$

In the above equations

$$
\Delta=s S\left(\alpha^{2}-\beta^{2}\right)-2 \alpha \beta(1-c C)
$$

and

$$
C=\cosh (\beta L), \quad c=\cos (\alpha L), \quad S=\sinh (\beta L) \quad \text { and } \quad s=\sin (\alpha L)
$$

The elements of the dynamic stiffness matrix are frequency dependent complex quantities because $\alpha$ and $\beta$ are functions of $\omega$ and damping factors.

So far we did not explicitly consider any forces within the element. A distributed body force can be considered following the usual finite element approach [52] for the 
classical finite element method, and replacing the static shape functions with the dynamic shape functions given in Eq. (38). Suppose $p(x, \omega), x \in[0, L]$ is the frequency depended distributed body force. The element nodal forcing vector can be obtained as

$$
\mathbf{f}(\omega)=\int_{0}^{L} p(x, \omega) \mathbf{N}(x, \omega) \mathrm{d} x
$$

As an example, if a point harmonic force of magnitude $p$ is applied at length $b<L$ then, $p(x, \omega)=p \delta(x-b)$ where $\delta(\bullet)$ is the Dirac delta function. The element nodal force vector becomes

$$
\mathbf{f}(\omega)=p \int_{0}^{L} \delta(x-b) \mathbf{N}(x, \omega) \mathrm{d} x
$$

For a structure built up from beam members with different properties, the dynamic stiffness matrices of Eq. (44) for all members can then be easily assembled as in the classical finite element method. This will lead to the global dynamic stiffness matrix $\mathbf{D}_{g}(\omega)$ for the whole structure, whose dynamic equilibrium can be also expressed by

$$
\mathbf{D}_{g}(\omega) \widehat{\mathbf{v}}_{g}(\omega)=\widehat{\mathbf{f}}_{g}(\omega)
$$

This equation can be directly used for the forced vibration analysis. For the free vibration analysis, natural frequencies $\omega$ are solved for when $\mathbf{D}_{g}(\omega) \widehat{\mathbf{v}}_{g}(\omega)=\mathbf{0}$ has non-trivial solutions and will be mentioned later in Section 4.

It is worth emphasising that for both the free and forced vibration analysis of a beam member with a uniform cross-section, the dynamic finite element method only needs a $4 \times 4$ matrix as in Eq. (48) with complex entries whereas the classical finite element method in Eq. (35) needs a number of elements which depends on the frequency range and desired analysis accuracy. This is a significant advantage of the proposed dynamic finite element approach compared to the conventional finite element approach discussed in the previous subsection.

\subsection{Analysis of the dynamic stiffness matrix}

The dynamic stiffness matrix given by Eq. (48) is general and expressed in a compact form. There are six unique coefficients. Here we discuss three special cases for the undamped system so that Eq. (48) becomes intuitively meaningful.

First we consider the special case when there is no elastic foundation and the frequency is zero, that the static case. When $k=0$ and $\omega \rightarrow 0$, from Eqs. (17) and (18) we have $\alpha \rightarrow 0$ and $\beta \rightarrow 0$. Note that the damping has no influence when the frequency is 0 . Considering this case and taking the mathematical limit we have

$$
\lim _{\alpha, \beta \rightarrow 0} \mathbf{D}(\omega)=\mathbf{D}_{0}=\frac{E I}{L^{3}}\left[\begin{array}{cccc}
12 & 6 L & -12 & 6 L \\
6 L & 4 L^{2} & -6 L & 2 L^{2} \\
-12 & -6 L & 12 & -6 L^{2} \\
6 L & 2 L^{2} & -6 L & 4 L^{2}
\end{array}\right]
$$

This matrix is the well known static stiffness matrix of a conventional (local) EulerBernoulli beam without an elastic foundation. This demonstrates that the static stiffness matrix is a special case of the general dynamic stiffness matrix given in Eq. (48).

Next we consider the case when the frequency is zero but the foundation stiffness is not zero, that is $\omega \rightarrow 0$ and $k \neq 0$. Neglecting the nonlocal effect, the general dynamic 
stiffness matrix degenerates to the following special case

$$
\begin{aligned}
& \lim _{\omega,\left(e_{0} a\right) \rightarrow 0} \mathbf{D}(\omega)=\mathbf{D}_{1} \\
& =\frac{2 E I b^{\prime}}{\left(S^{2}-s^{2}\right)}\left[\begin{array}{cccc}
2{b^{\prime}}^{2}(S C+s c) & b^{\prime}\left(C^{2}-c^{2}\right) & -2{b^{\prime}}^{2}(c S+s C) & 2 s S b^{\prime} \\
b^{\prime}\left(C^{2}-c^{2}\right) & S C-s c & -2 s S b^{\prime} & s C-c S \\
-2{b^{\prime}}^{2}(c S+s C) & -2 s S b^{\prime} & 2{b^{\prime 2}}^{2}(S C+s c) & -b^{\prime}\left(C^{2}-c^{2}\right) \\
2 s S b^{\prime} & s C-c S & -b^{\prime}\left(C^{2}-c^{2}\right) & S C-s c
\end{array}\right]
\end{aligned}
$$

In the above equation

$$
b^{\prime}=\sqrt[4]{\frac{k}{4 E I}}
$$

and

$$
C=\cosh \left(b^{\prime} L\right), \quad c=\cos \left(b^{\prime} L\right), \quad S=\sinh \left(b^{\prime} L\right) \quad \text { and } \quad s=\sin \left(b^{\prime} L\right)
$$

Equation (61) represents the exact transcendental stiffness matrix of a conventional (local) Euler-Bernoulli beam resting on an elastic foundation (see for example [27]). To understand the nature of this transcendental stiffness matrix further, it is expanded in a Taylor series about $k=0$. Substituting $b^{\prime}$ from equation (62) in equation (61) and differentiating all the elements with respect to $k$, after simplifications we have the series expansion

$$
\begin{aligned}
\mathbf{D}_{1} & =\frac{E I}{L^{3}}\left[\begin{array}{cccc}
12 & 6 L & -12 & 6 L \\
6 L & 4 L^{2} & -6 L & 2 L^{2} \\
-12 & -6 L & 12 & -6 L^{2} \\
6 L & 2 L^{2} & -6 L & 4 L^{2}
\end{array}\right]+\frac{L}{420}\left[\begin{array}{cccc}
156 & 22 L & 54 & -13 L \\
22 L & 4 L^{2} & 13 L & -3 L^{2} \\
54 & 13 L & 156 & -22 L \\
-13 L & -3 L^{2} & -22 L & 4 L^{2}
\end{array}\right] k \\
& +\frac{L^{5}}{69854400 E I}\left[\begin{array}{cccc}
-25488 & -5352 L & -23022 & 5043 L \\
-5352 L & -1136 L^{2} & -5043 L & 1097 L^{2} \\
-23022 & -5043 L & -25488 & 5352 L \\
5043 L & 1097 L^{2} & 5352 L & -1136 L^{2}
\end{array}\right] k^{2}+O\left(k^{3}\right)+\cdots
\end{aligned}
$$

It is interesting to note that the first two terms of this expansion is exactly the same as the first two terms of the static nonlocal matrix in equation (33).

For the third case we consider the special condition when the beam is a local beam. In that case $e_{0} a=0$ and consequently

$$
\alpha=\beta=b=\sqrt[4]{\frac{m \omega^{2}-k}{E I}}
$$

Using these, the general dynamic stiffness matrix degenerates to the following special case

$$
\lim _{\alpha \rightarrow b, \beta \rightarrow b} \mathbf{D}(\omega)=\mathbf{D}_{2}(\omega)=\frac{E I b}{C c-1}\left[\begin{array}{cccc}
-\beta^{2}(C s+S c) & -\beta S s & \beta^{2}(S+s) & -\beta(C-c) \\
-\beta S s & -C s+S c & \beta(C-c) & -S+s \\
\beta^{2}(S+s) & \beta(C-c) & -\beta^{2}(C s+S c) & \beta S s \\
-\beta(C-c) & -S+s & \beta S s & -C s+S c
\end{array}\right]
$$

here

$$
C=\cosh (b L), \quad c=\cos (b L), \quad S=\sinh (b L) \quad \text { and } \quad s=\sin (b L)
$$

The equation derived in (66) match exactly with the dynamic stiffness matrix of a con- 
ventional (local) Euler-Bernoulli beam without an elastic foundation [55]. It is useful to expand the dynamic stiffness matrix in (66) in a Taylor series around $\omega=0$. Ignoring the foundation stiffness $(k=0)$ and after some simplification we can deduce that

$$
\begin{aligned}
\mathbf{D}_{2}(\omega) & =\frac{E I}{L^{3}}\left[\begin{array}{cccc}
12 & 6 L & -12 & 6 L \\
6 L & 4 L^{2} & -6 L & 2 L^{2} \\
-12 & -6 L & 12 & -6 L^{2} \\
6 L & 2 L^{2} & -6 L & 4 L^{2}
\end{array}\right]-\omega^{2} \frac{m L}{420}\left[\begin{array}{cccc}
156 & 22 L & 54 & -13 L \\
22 L & 4 L^{2} & 13 L & -3 L^{2} \\
54 & 13 L & 156 & -22 L \\
-13 L & -3 L^{2} & -22 L & 4 L^{2}
\end{array}\right] \\
& -\omega^{4} \frac{m^{2} L^{6}}{161700 E I}\left[\begin{array}{cccc}
\frac{59}{L} & \frac{223}{18} & \frac{1279}{24 L} & -\frac{1681}{144} \\
\frac{223}{18} & \frac{71 L}{27} & \frac{1681}{144} & -\frac{1097 L}{432} \\
\frac{1279}{24 L} & \frac{1681}{144} & \frac{59}{L} & -\frac{223}{18} \\
-\frac{1681}{144} & -\frac{1097 L}{432} & -\frac{223}{18} & \frac{71 L}{27}
\end{array}\right]+O\left(\omega^{6}\right)+\cdots
\end{aligned}
$$

It is useful to note that the first two terms of this expansion is exactly the same as the stiffness and mass matrix of a classical Euler-Bernoulli beam. A similar Taylor series expansion in $\omega$ can also be performed for the general dynamic stiffness matrix in (48). This analysis clearly shows that the dynamic stiffness matrix contains the static stiffness matrix and the mass matrix and also contains all the higher orders terms associated with frequency. For this reason, Eq. (48) is valid for all values of frequency.

\section{Free vibration analysis of nonlocal beams}

In the last section, two forms of dynamic stiffness matrices have been developed based on both the classical finite element method (Subsection 3.1) and dynamic finite element method (Subsection 3.2). The corresponding procedures for free vibration analyses are provided as follows.

For the nonlocal beam element based on the classical finite element method as discussed in Subsection 3.1, the stiffness matrix $\mathbf{K}_{e}$ of Eq. (33) and mass matrix $\mathbf{M}_{e}$ of Eq. (34) are frequency independent. Therefore, after assembling elemental matrices $\left(\mathbf{K}_{e}\right.$ and $\mathbf{M}_{e}$ ) into the corresponding global matrices $\left(\mathbf{K}_{g}\right.$ and $\left.\mathbf{M}_{g}\right)$ and applying prescribed boundary conditions, one will arrive at a generalized eigenvalue problem $\left(\mathbf{K}_{g}-\omega^{2} \mathbf{M}_{g}\right) \hat{\mathbf{v}}_{e}(\omega)=\hat{\mathbf{0}}$, where the natural frequencies can be computed by using well-established linear algebraic solver.

For the nonlocal beam member based on dynamic finite element method (Section 3.2), after assembling procedure and application of prescribed boundary conditions such as in the classical finite element method, one will obtain the global dynamic stiffness matrix $\mathbf{D}_{g}(\omega)$. Since each nonzero entry of the matrix $\mathbf{D}_{g}(\omega)$ is a transcendental functions of frequency $\omega$ and nonlocal parameter $e_{0} a$, a special solution technique called the WittrickWilliams algorithm [56] need to applied to compute the natural frequencies $\omega$. Eq. (69) is the key equation of the Wittrick-Williams algorithm, which is used to calculate the mode count $J$ when $\omega$ is lower than the trial frequency $\omega^{*}$

$$
J\left(\omega^{*}\right)=\sum_{i=1}^{N_{e}} J_{0 i}\left(\omega^{*}\right)+s\left\{\mathbf{D}_{g}\left(\omega^{*}\right)\right\}
$$

where $s\left\{\mathbf{D}_{g}\left(\omega^{*}\right)\right\}$ is the number of negative diagonal elements after upper triangular transformation by using Gauss elimination of $\mathbf{D}_{g}\left(\omega^{*}\right)$ when $\omega=\omega^{*} . J_{0 i}\left(\omega^{*}\right)$ is the number 
of natural frequencies between $\omega=0$ and $\omega=\omega^{*}$ when both ends of the $i$ th beam member are clamped and $N_{e}$ is the number of beam members.

Undoubtedly, $J_{0 i}\left(\omega^{*}\right)$ plays an important role in the Wittrick-Williams algorithm. There are three ways of getting the value of $J_{0 i}\left(\omega^{*}\right)$ for the $i$ th member.

The first technique is to refine the mesh to make sure $J_{0 i}=0$. Obviously, it will introduce unnecessary computational cost significantly.

The second technique is letting the common denominator of the member's dynamic stiffness matrix (see Eq. (55)) become zero, i.e., $s S\left(\alpha^{2}-\beta^{2}\right)-2 \alpha \beta(1-c C)=0$. Then the natural frequencies $\omega$ s can be numerically solved and $J_{0 i}\left(\omega^{*}\right)$ can be easily obtained. However, this technique relies on numerical solvers for transcendental equations and might miss some of the roots.

A third technique is applied in this study which is the most reliable and efficient, i.e., the $J_{0 i}$ problem of a beam member is resolved by applying an indirect method [57], which improves the computational efficiency of the dynamic stiffness method. According to Wittrick-Williams algorithm, the mode count $J_{S S i}\left(\omega^{*}\right)$ of the $i$ th beam member with both ends simply supported can be given by Eq. (69), which can be recast as $J_{S S i}\left(\omega^{*}\right)=$ $J_{0 i}\left(\omega^{*}\right)+s\left\{\mathbf{D}_{S S i}\left(\omega^{*}\right)\right\}$, where $\mathbf{D}_{S S i}\left(\omega^{*}\right)$ is the dynamic stiffness matrix for the $i$ th beam member with both ends simply supported. Therefore

$$
J_{0 i}\left(\omega^{*}\right)=J_{S S i}\left(\omega^{*}\right)-s\left\{\mathbf{D}_{S S i}\left(\omega^{*}\right)\right\}
$$

Next we will provide the analytical expression for both $J_{S S i}\left(\omega^{*}\right)$ and $s\left\{\mathbf{D}_{S S i}\left(\omega^{*}\right)\right\}$. For a simply-supported nonlocal beam member with length $L$, the shape function can be given as $v(x)=C \sin (j \pi / L)$ where $j$ is a non-negative integer and $C$ is an arbitrary constant. Substituting into Eq. (27) and ignoring the damping effect, one will have

$$
(j \pi / L)^{2}=\bar{b}^{2}\left(\sqrt{4+\gamma^{2}}+\gamma\right) / 2
$$

and solving for $J$ leads to

$$
J_{S S i}\left(\omega^{*}\right)=\text { floor }\left(\bar{b} L / \pi \sqrt{\left(\sqrt{4+\gamma^{2}}+\gamma\right) / 2}\right)
$$

where $\gamma=\bar{b}^{2}\left(e_{0} a\right)^{2}$, and $\bar{b}^{4}=\left(m \omega^{* 2}-k\right) / E I$ and floor gives the largest integer smaller than the followed value. Besides, the matrix $\mathbf{D}_{S S i}\left(\omega^{*}\right)$ for the $i$ th member is essentially the second and fourth rows and columns of the dynamic stiffness matrix in Eq. (48) after applying the simply supported boundary conditions on both ends, namely,

$$
\mathbf{D}_{S S i}\left(\omega^{*}\right)=E I\left[\begin{array}{ll}
d_{3} & d_{6} \\
d_{6} & d_{3}
\end{array}\right]
$$

Therefore,

$$
s\left\{\mathbf{D}_{S S i}\left(\omega^{*}\right)\right\}=s\left(d_{3}\right)+s\left(d_{3}-d_{6}^{2} / d_{3}\right)
$$

It is easy to know that for $s(\bullet)$ takes 1 when ' $\bullet$ ' is negative scalar and zero for nonnegative scalar. Next we illustrate the formulations derived in these two sections using an example. 


\section{Numerical results and discussions}

\subsection{Analysis of the dynamic stiffness coefficients}

The generality of the dynamic stiffness matrix given in Eq. (48) was investigated analytically in Subsection 3.3. Here we numerically investigate the scope of the dynamic stiffness matrix. There are six unique elements in the matrix. They are complex-valued and frequency-dependent quantities. A double-walled carbon nanotube (DWCNT) is considered to illustrate the dynamic stiffness matrix. An armchair $(5,5),(8,8)$ DWCNT with Young's modulus $E=1.0 \mathrm{TPa}, L=30 \mathrm{~nm}$, density $\rho=2.3 \times 10^{3} \mathrm{~kg} / \mathrm{m}^{3}$ and thickness $t=0.35 \mathrm{~nm}$ is considered as in [58]. The inner and the outer diameters of the DWCNT are respectively $0.68 \mathrm{~nm}$ and $1.1 \mathrm{~nm}$. The system considered here is shown in Fig. 3. In Fig. 3, we show the pinned-pinned boundary condition for the DWCNT as an

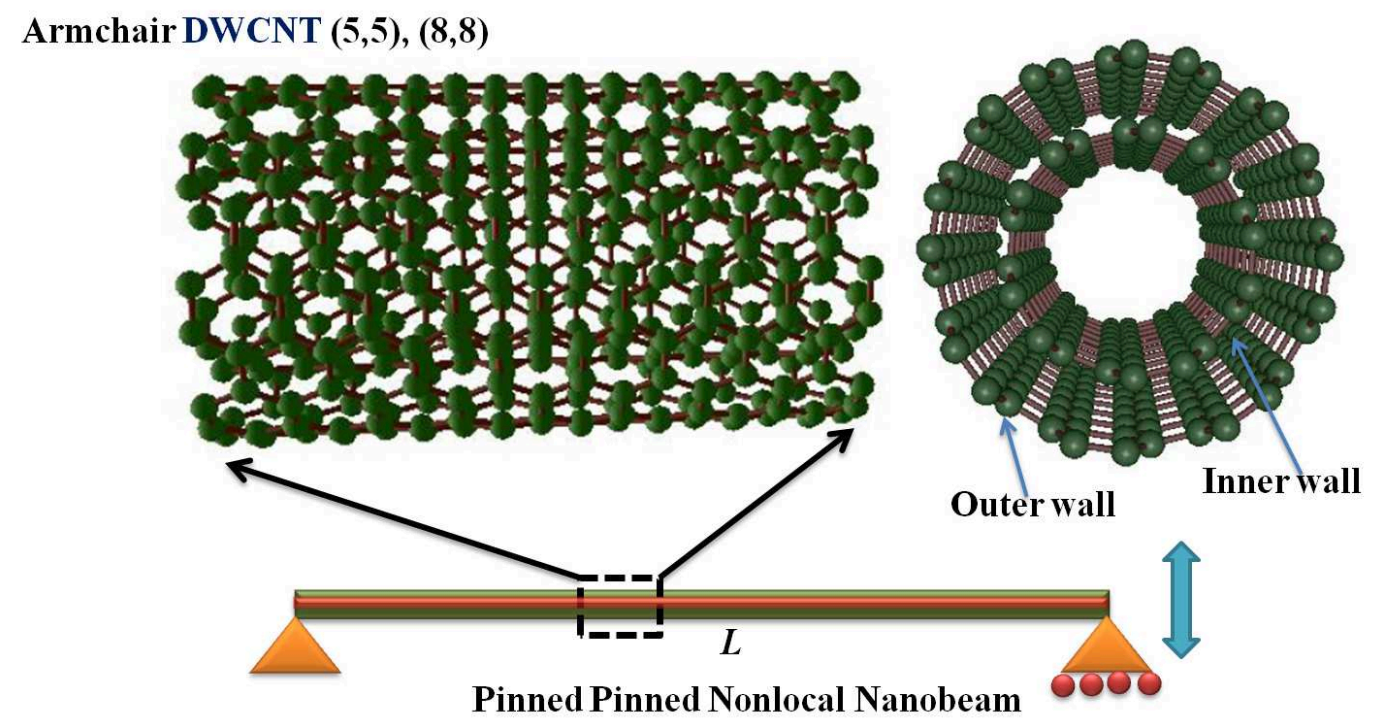

Fig. 3: Bending vibration of an armchair $(5,5),(8,8)$ double-walled carbon nanotube (DWCNT) with pinned-pinned boundary condition.

illustration. The coefficients of the dynamic stiffness matrix are not dependent on the boundary conditions.

In Fig. 4 we show the amplitudes of all the six unique dynamic stiffness coefficients of the DWCNT for $e_{0} a=0.5 \mathrm{~nm}$. The stiffness and mass proportional damping factors are $\zeta_{1}=10^{-4}$ and $\zeta_{2}=0.05$ respectively. Each dynamic stiffness coefficient is normalised with respect to its static value given in (60). Although the dynamic coefficients are different in values, they have peaks at the same frequency. These peaks do not correspond to the resonance frequency of the beam (they will depend on the boundary conditions). The peak frequencies correspond to the real part of the complex solution of the denominator equation (55) given by

$$
\Delta=0 \quad \text { or } \quad s S\left(\alpha^{2}-\beta^{2}\right)=2 \alpha \beta(1-c C)
$$

The frequency axis is normalised with respect to the first natural frequency of a pinnedpinned local Euler-Bernoulli beam, that is

$$
\omega_{1}=\left(\frac{\pi}{L}\right)^{2} \sqrt{\frac{E I}{m}}
$$




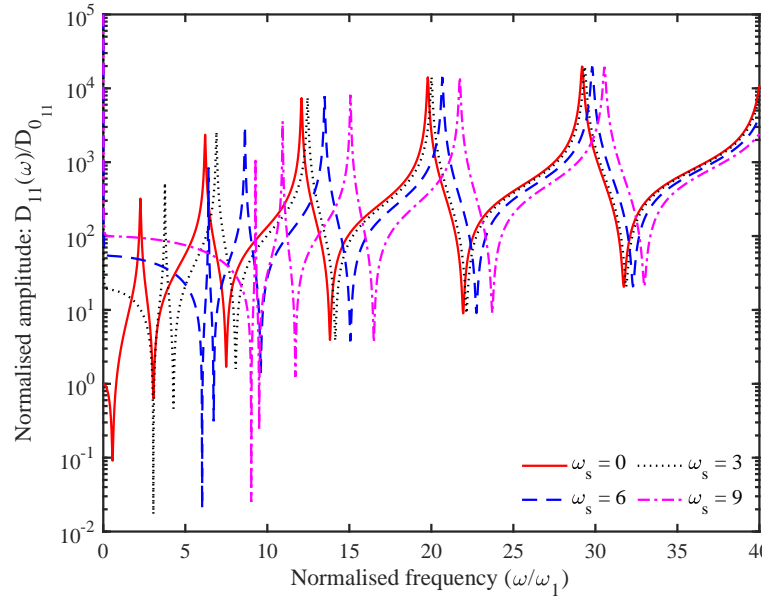

(a) $D_{11}(\omega)$

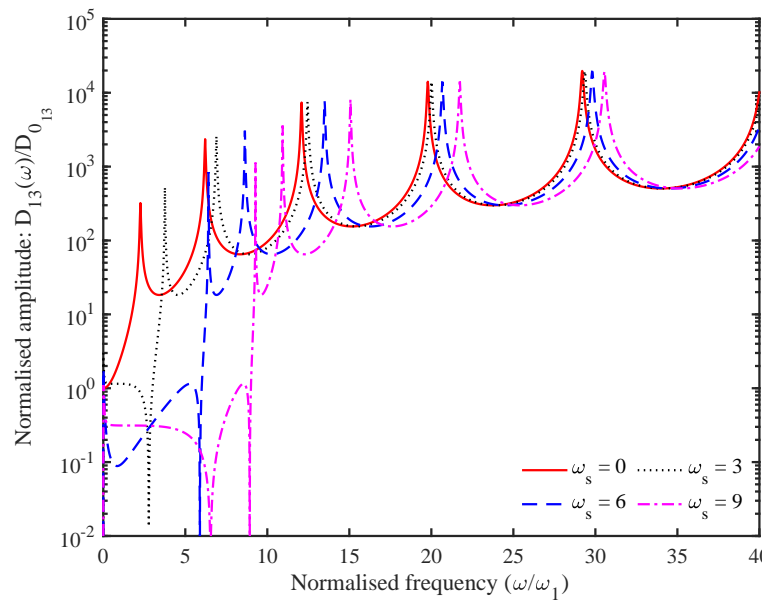

(c) $D_{13}(\omega)$

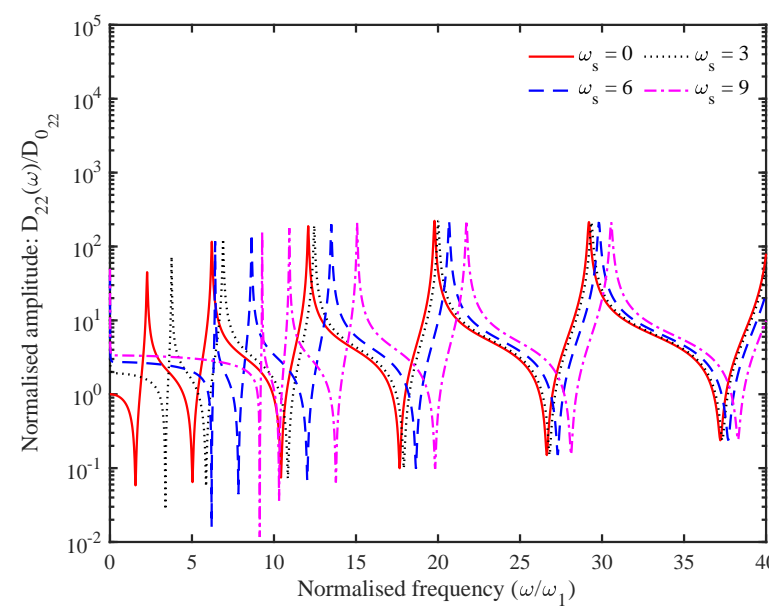

(e) $D_{22}(\omega)$

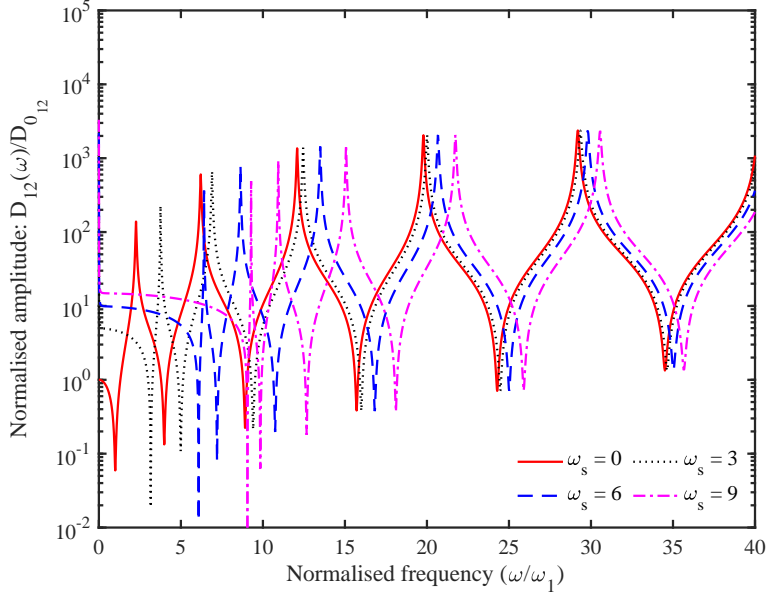

(b) $D_{12}(\omega)$

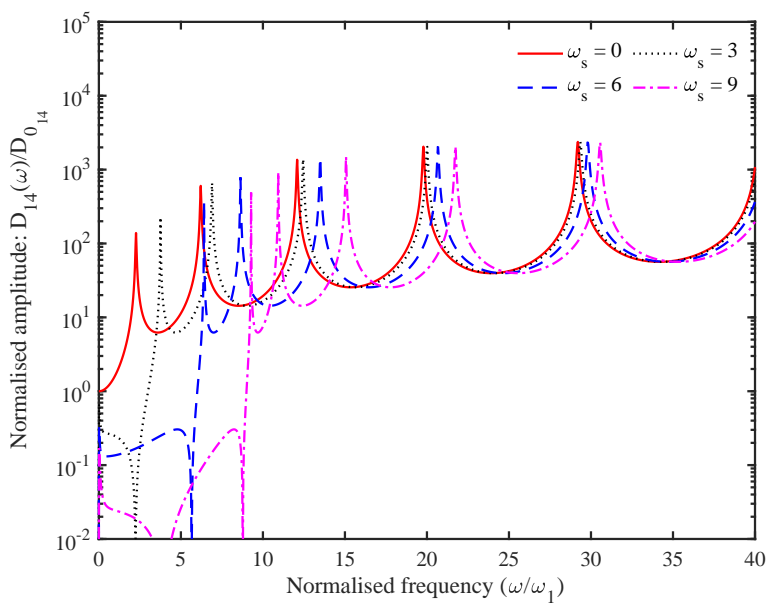

(d) $D_{14}(\omega)$

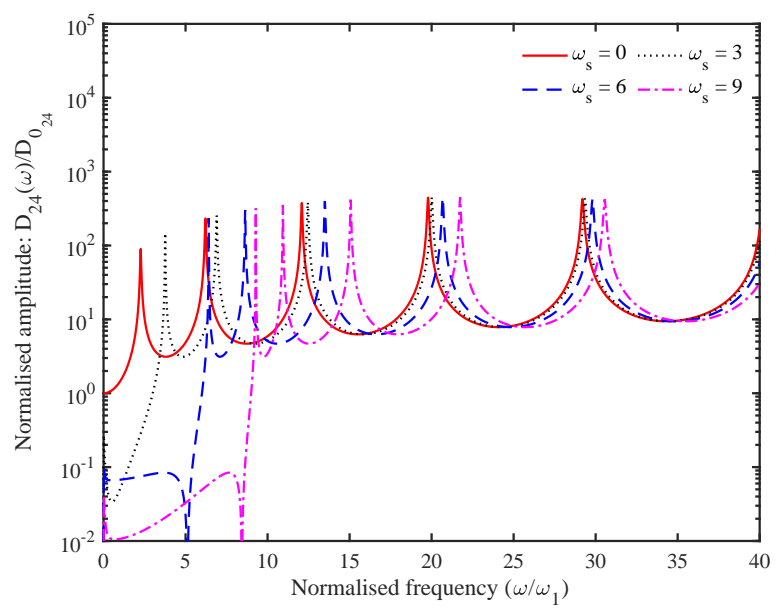

(f) $D_{24}(\omega)$

Fig. 4: Amplitudes of the six unique dynamic stiffness coefficients of the DWCNT for $e_{0} a=0.5 \mathrm{~nm}$. The stiffness and mass proportional damping factors are $\zeta_{1}=10^{-4}$ and $\zeta_{2}=0.05$ respectively. In each plot, we have shown four values of foundation stiffness $k=\omega_{s}^{2} m$. Each dynamic stiffness coefficient is normalised with respect to its static value given in Eq. (60). 
In each plot, four values of the foundation stiffness obtained as $k=\omega_{s}^{2} m$ are shown. The case when $\omega_{s}=0$ represents when there is no elastic foundation. This case represents the lowest peaks in the dynamic stiffness coefficients. Higher values of foundation stiffness $k$ move the peaks to the right, indicating that the system is becoming stiffer. The foundation stiffness has a higher impact on the first peak. Its effect diminishes in the higher frequencies. Amplitudes of the six unique dynamic stiffness coefficients of the DWCNT for $e_{0} a=1.5 \mathrm{~nm}$ are shown in Fig. 5. This is similar to the previous plot, except for a higher value of the nonlocal parameter. We have shown four values of foundation stiffness $k=\omega_{s}^{2} m$. A key difference between this case from Fig. 4 is that there is more number of peaks within the chosen frequency range. This is because a higher value of the nonlocal parameter reduces the peak frequency values. We also observe that the foundation stiffness moves the fist peak significantly higher. As an example, for $\omega_{s}=9$, the first peak is close to the third peak of the beam without the elastic foundation. Such extreme difference is less pronounced in the higher frequency regions.

Figures 4 and 5 clarify the individual variation of the six unique dynamic stiffness coefficients as a function of frequency. Now we are interested on how the coefficients compare and contrast to each other. In Fig. 6 amplitudes of the normalised dynamic stiffness coefficients are shown for $e_{0} a=1.0 \mathrm{~nm}$. Four different values of the foundation stiffness $k=\omega_{s}^{2} m$ are shown in the four subplots. The dynamic stiffness coefficients are normalised with respect to their respective static values given in Eq. (60). All the six coefficients start with a value of 1 in Fig. 6 (a) confirming the validity of the static limit in Eq. (60). The plots in Fig. 6 reconfirm that even the amplitudes of the dynamic stiffness coefficients are different, they all peak at the same frequency values, which arise from the solution of denominator equation (75). From Fig. 6(b), (c) and (d) we also observe that higher values of the foundation stiffness progressively shift the peak towards higher frequency ranges. Amplitudes of the dynamic stiffness coefficients for a higher value of the nonlocal parameter, namely $e_{0} a=1.5 \mathrm{~nm}$, are shown in Fig. 7. Here we can clearly see more number of peaks compared to Fig. 6 within the same frequency range. This in turn also implies that the spacing between the peaks is comparatively smaller.

In the discussions so far, the damping factor values are kept constant. Now we investigate the role of damping factors on the dynamic stiffness coefficients. In Fig. 8 amplitudes of all the six dynamic stiffness coefficients are shown. The values of the nonlocal parameter and the foundation stiffness are kept constant with $e_{0} a=1.0 \mathrm{~nm}$ and $\omega_{s}=3$. Six combinations of damping factor values consisting $\zeta_{1}=10^{-2}, 10^{-3}, 10^{-4}$ and $\zeta_{2}=0.05,0.1$ are shown. The stiffness proportional damping factor $\zeta_{1}$ has the most impact on the dynamic stiffness coefficients in the high-frequency ranges. For higher values of $\zeta_{1}$, the dynamic stiffness coefficients are independent of the $\zeta_{2}$ values. On the other hand, for smaller values of $\zeta_{1}$, the amplitudes of dynamic stiffness coefficients depend on the mass proportional damping factor $\zeta_{2}$. As expected, smaller damping factor values lead to higher peaks in the dynamic stiffness coefficients.

\subsection{Dynamic response analysis in the frequency domain}

Results in the previous section exposed the nature of the coefficients of the proposed dynamic stiffness matrix. In the presence of damping, they are complex valued functions of the frequency. In this subsection we demonstrate how to utilise the nonlocal dynamic stiffness matrix for nano-mechanical systems. In Fig. 3 the DWCNT is shown with a 


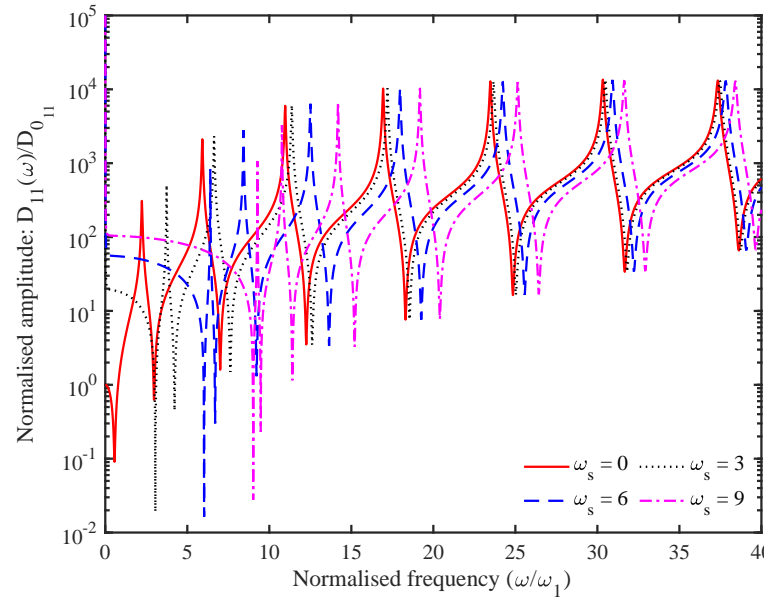

(a) $D_{11}(\omega)$

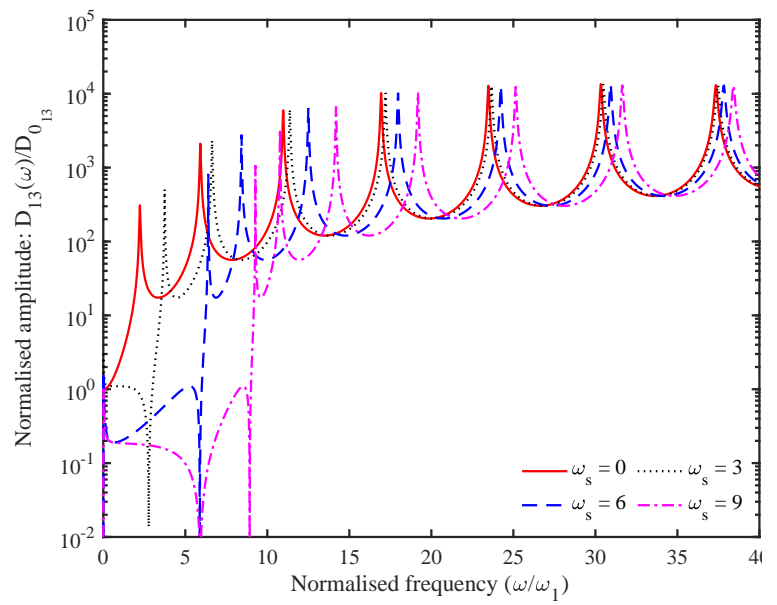

(c) $D_{13}(\omega)$

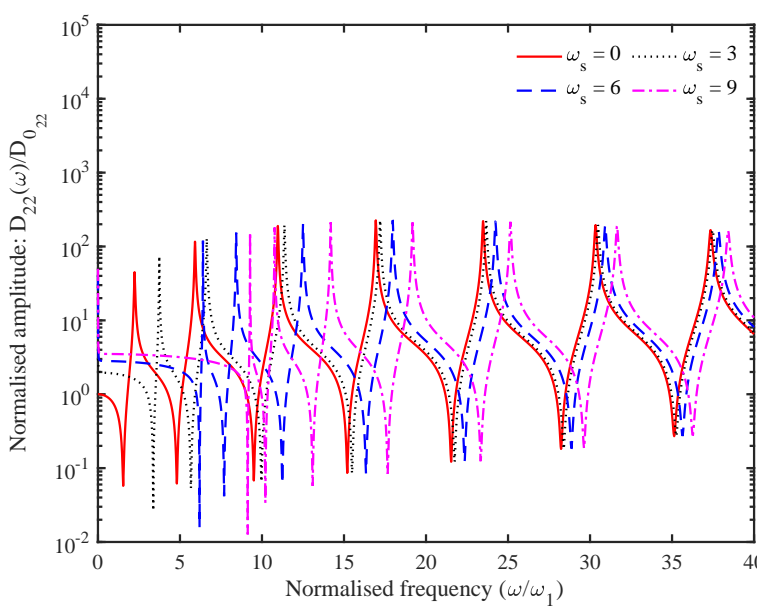

(e) $D_{22}(\omega)$

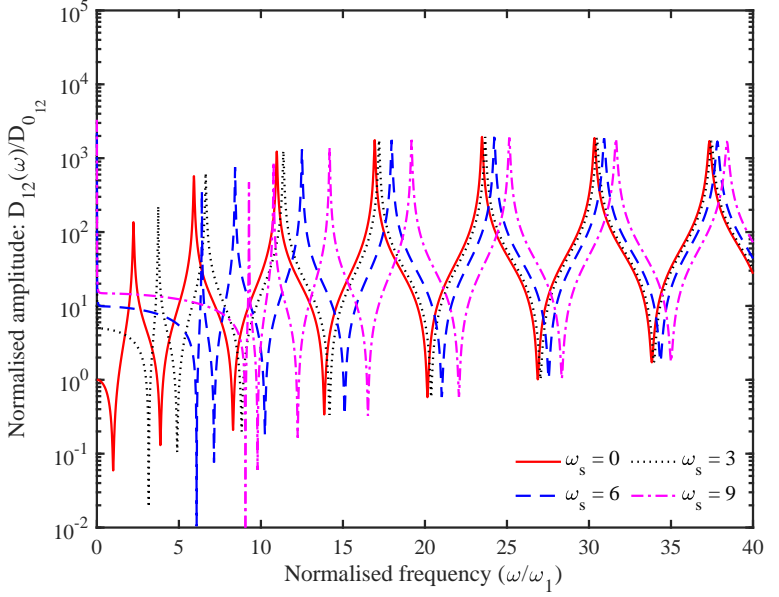

(b) $D_{12}(\omega)$

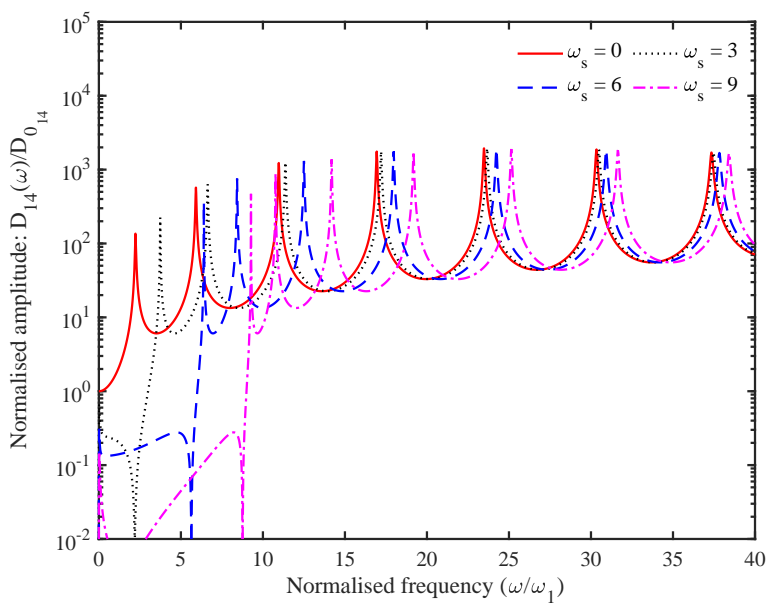

(d) $D_{14}(\omega)$

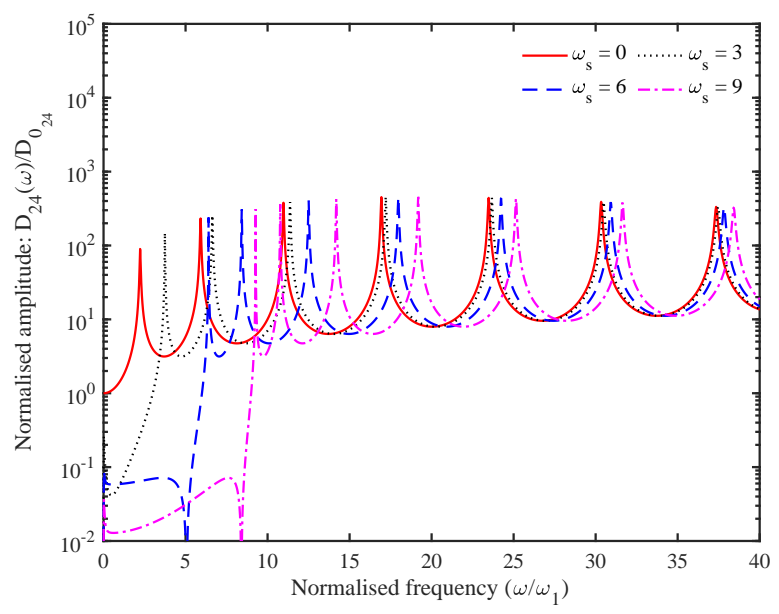

(f) $D_{24}(\omega)$

Fig. 5: Amplitudes of the six unique dynamic stiffness coefficients of the DWCNT for $e_{0} a=1.5 \mathrm{~nm}$. In each plot, we have shown four values of foundation stiffness $k=\omega_{s}^{2} m$. Each dynamic stiffness coefficient is normalised with respect to its static value given in Eq. (60). The stiffness and mass proportional damping factors are $\zeta_{1}=10^{-4}$ and $\zeta_{2}=0.05$ respectively. 


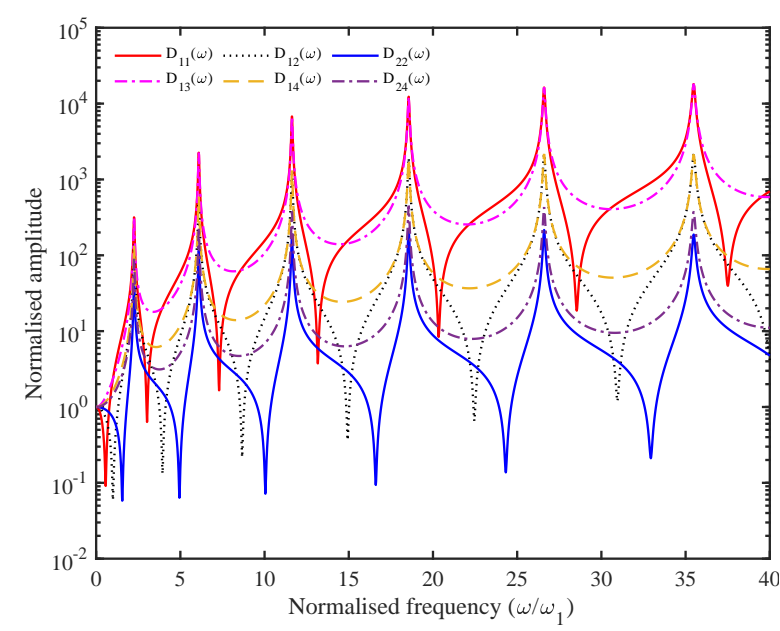

(a) $\omega_{s}=0$ (no elastic foundation)

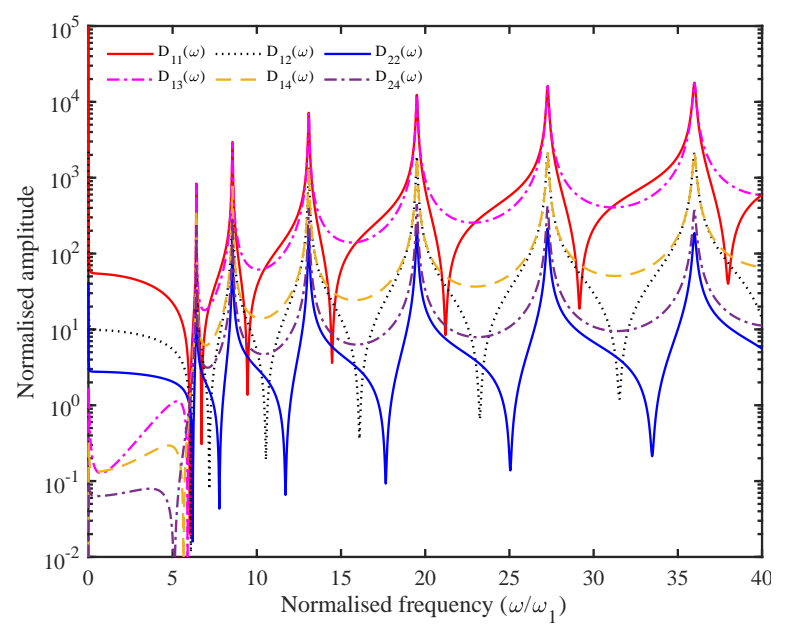

(c) $\omega_{s}=6.0$

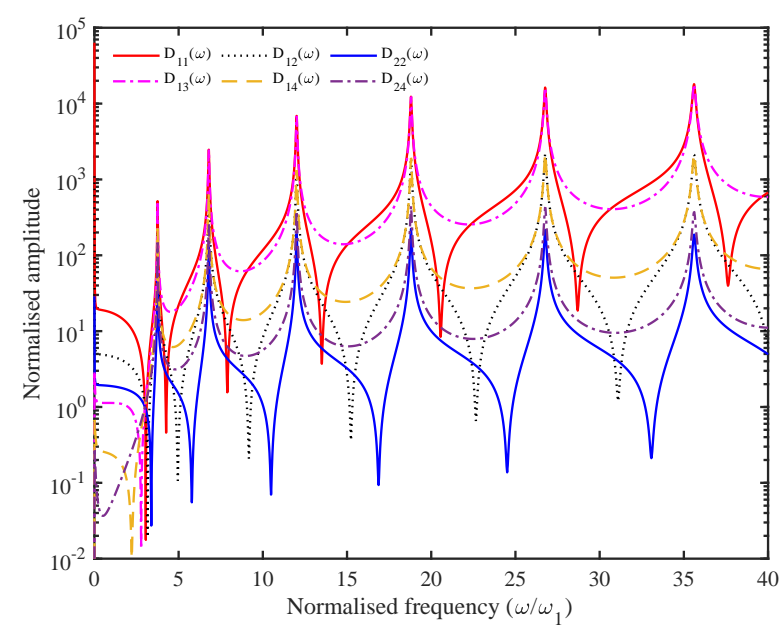

(b) $\omega_{s}=3.0$

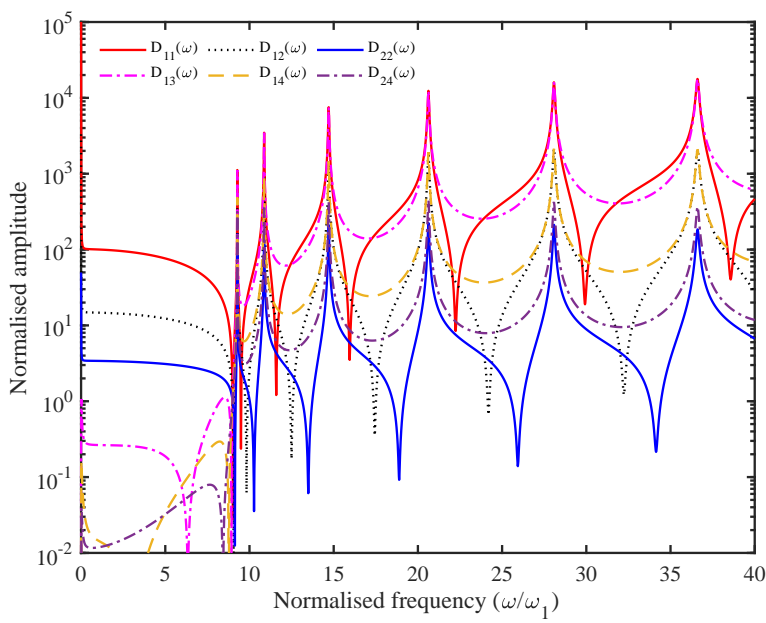

(d) $\omega_{s}=9.0$

Fig. 6: Amplitudes of the normalised dynamic stiffness coefficients of the DWCNT for $e_{0} a=1.0 \mathrm{~nm}$. The stiffness and mass proportional damping factors are $\zeta_{1}=10^{-4}$ and $\zeta_{2}=0.05$ respectively. In each plot, we have shown all the six unique dynamic stiffness coefficients together (normalised with respect to their respective static values). Four different values of foundation stiffness $k=\omega_{s}^{2} m$ are shown.

pinned-pinned boundary condition. Undamped nonlocal natural frequencies for this case can be obtained [59] as

$$
\lambda_{j}=\sqrt{\frac{E I}{m}} \frac{\beta_{j}^{2}}{\sqrt{1+\beta_{j}^{2}\left(e_{0} a\right)^{2}}} \text { where } \beta_{j}=j \pi / L, \quad j=1,2, \cdots
$$

Here $E I$ is the bending rigidity and $m$ is the mass per unit length of the DWCNT. For the finite element analysis, the DWCNT is divided into 100 elements. The dimension of each of the system matrices become $200 \times 200$, that is $n=200$. The global stiffness and mass matrices are obtained by assembling the element stiffness and mass matrix given by (33) and (34).

The natural frequencies obtained using the analytical expression (77) are compared with direct finite element simulation in Fig. 9. The frequency values are normalised with 


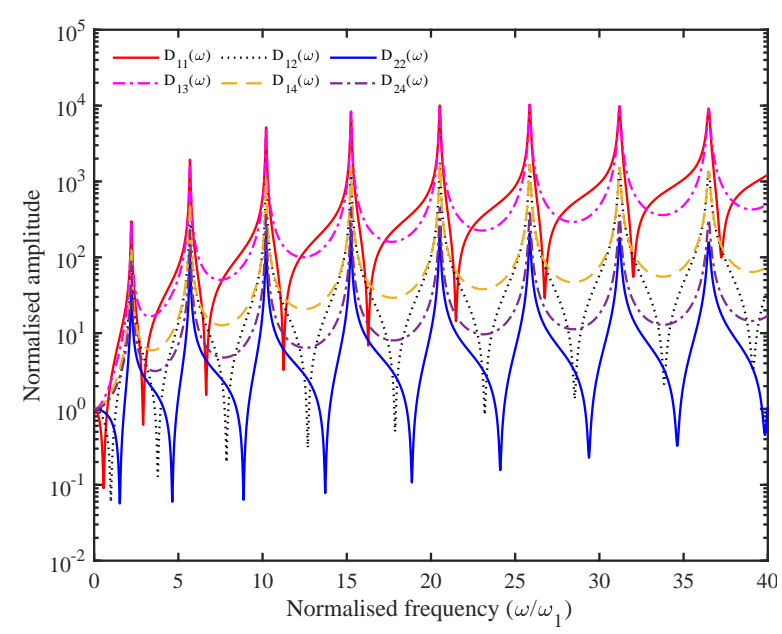

(a) $\omega_{s}=0$ (no elastic foundation)

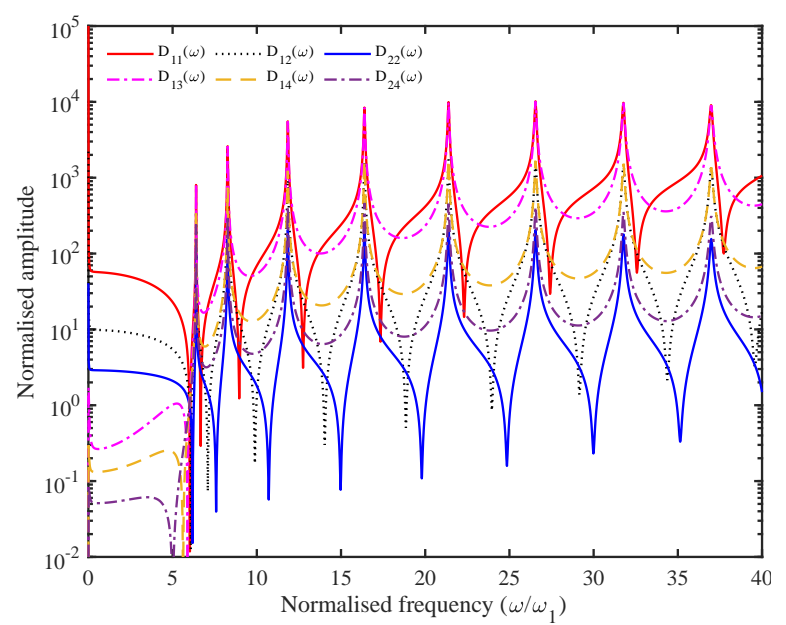

(c) $\omega_{s}=6.0$

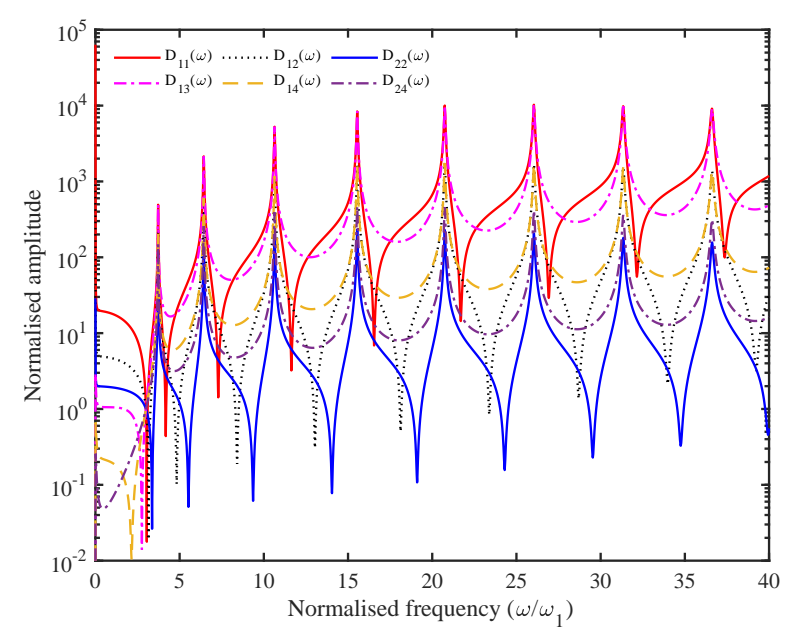

(b) $\omega_{s}=3.0$

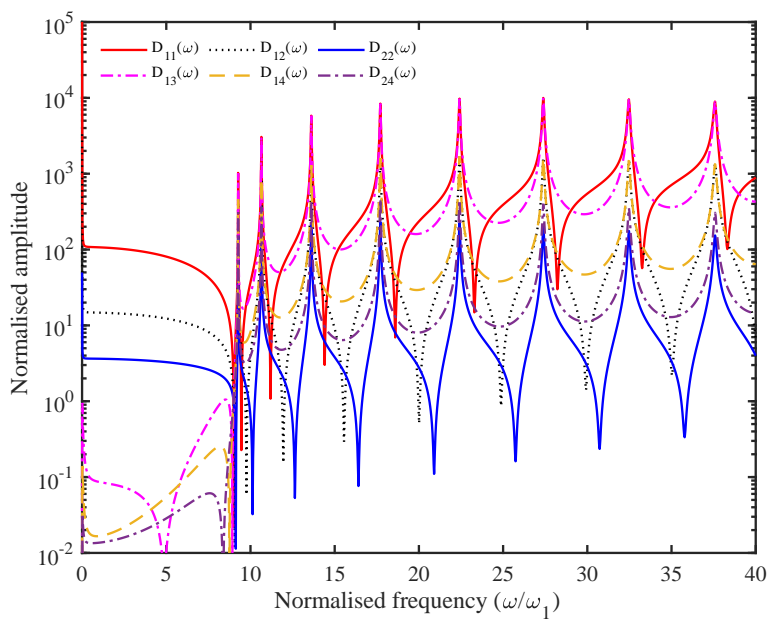

(d) $\omega_{s}=9.0$

Fig. 7: Amplitudes of the normalised dynamic stiffness coefficients of the DWCNT for $e_{0} a=2.0 \mathrm{~nm}$. In each plot, we have shown all the six unique dynamic stiffness coefficients together (normalised with respect to their respective static values). Four different values of foundation stiffness $k=\omega_{s}^{2} m$ are shown. The stiffness and mass proportional damping factors are $\zeta_{1}=10^{-4}$ and $\zeta_{2}=0.05$ respectively.

respect to the first local natural frequency. First 20 nonlocal natural frequencies without the elastic foundation are shown for four distinct values of $e_{0} a$, namely 0.5, 1.0, 1.5 and 2.0nm. Natural frequencies corresponding to the underlying local system is shown in Fig. 9. Local frequencies are qualitatively different from nonlocal frequencies as it increases quadratically with the number of modes. Nonlocal frequencies, on the other hand, increases approximately linearly with the number of modes.

For the response analysis, we first consider the rotation response of the pinned-pinned beam due to the application of a dynamic moment. The moment is applied at the right end of the beam and we are interested in the dynamic response

$$
\theta(\omega)=\frac{d v(\omega)}{d x}
$$

at the right-end. Considering the dynamic equilibrium in Eq. (45) and applying the 


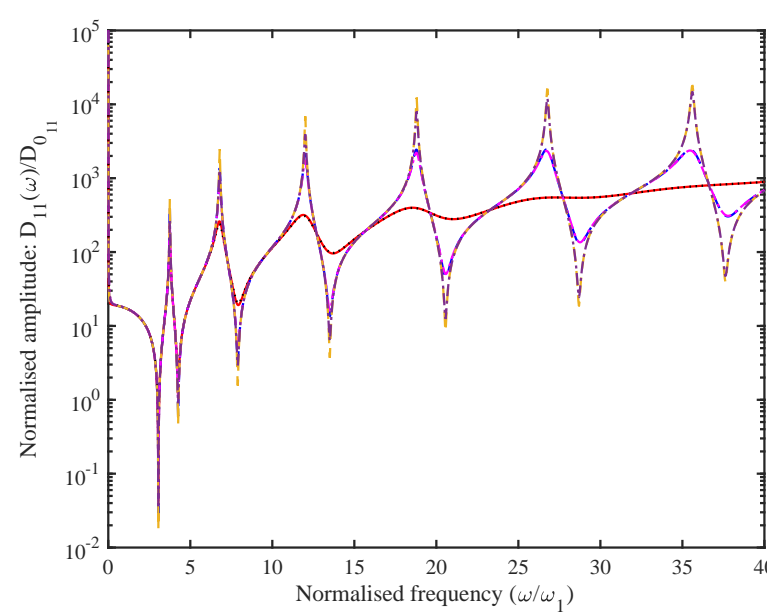

(a) $D_{11}(\omega)$

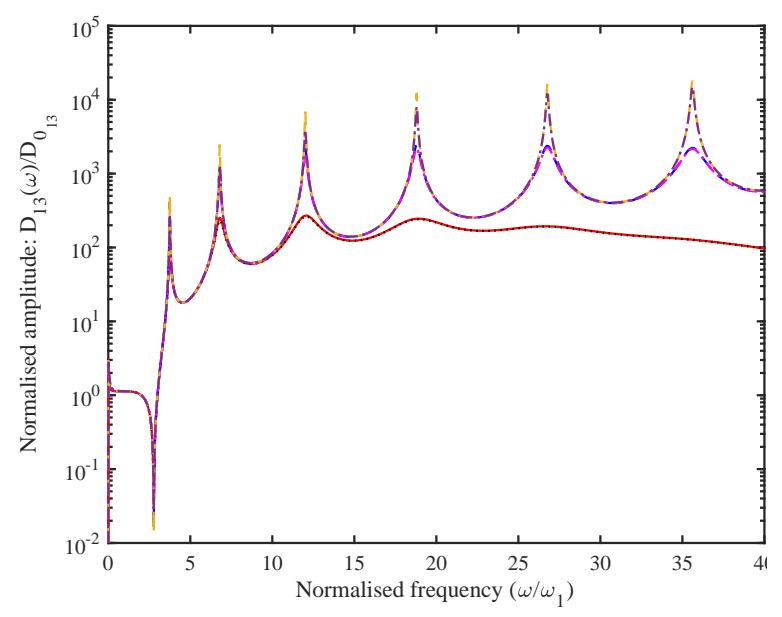

(c) $D_{13}(\omega)$

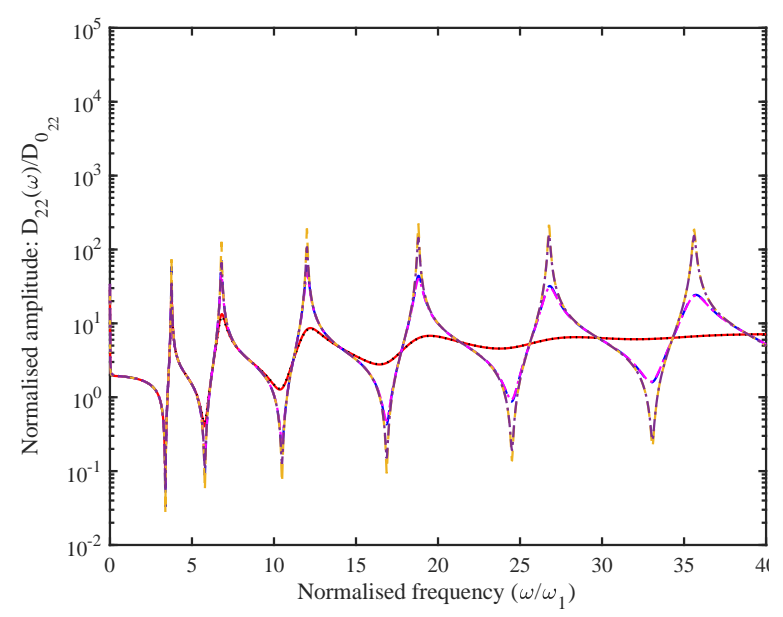

(e) $D_{22}(\omega)$

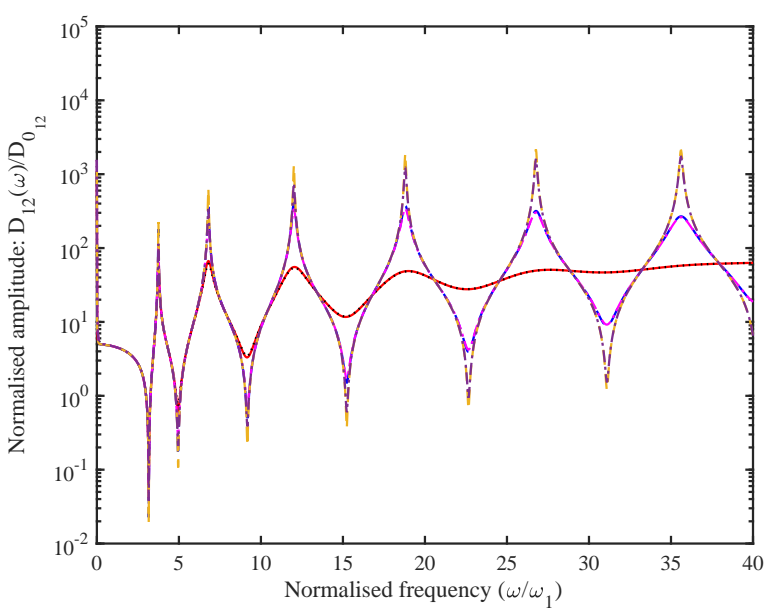

(b) $D_{12}(\omega)$

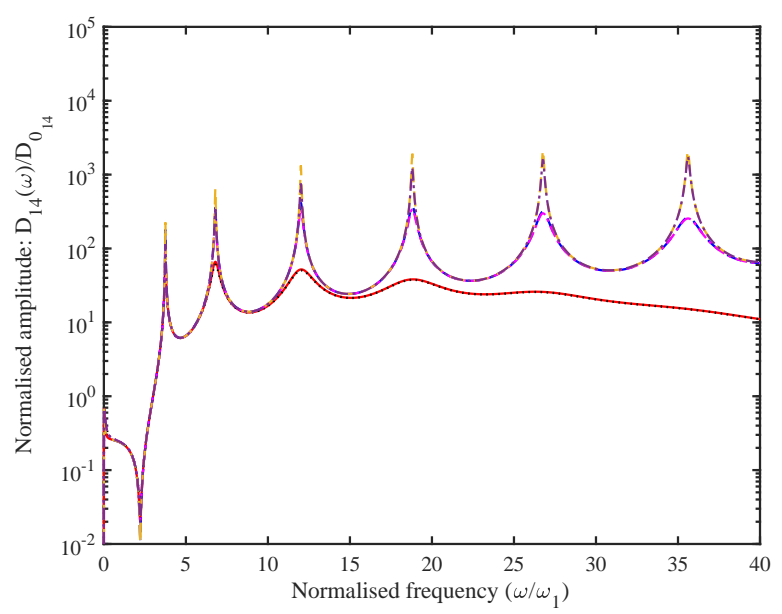

(d) $D_{14}(\omega)$

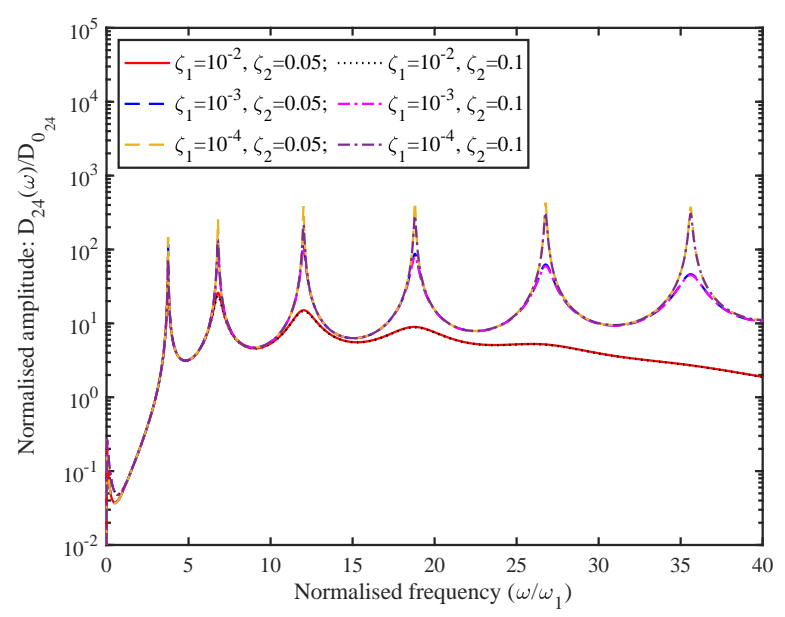

(f) $D_{24}(\omega)$

Fig. 8: Amplitudes of the six unique dynamic stiffness coefficients of the DWCNT for $e_{0} a=1 . \mathrm{nm}$ and $\omega_{s}=3$ (note that $k=\omega_{s}^{2} m$ ). In each plot, we have shown six combinations of damping factor values consisting $\zeta_{1}=10^{-2}, 10^{-3}, 10^{-4}$ and $\zeta_{2}=0.05,0.1$ (see the legend in (f)). Each dynamic stiffness coefficient is normalised with respect to its static value given in Eq. (60). 


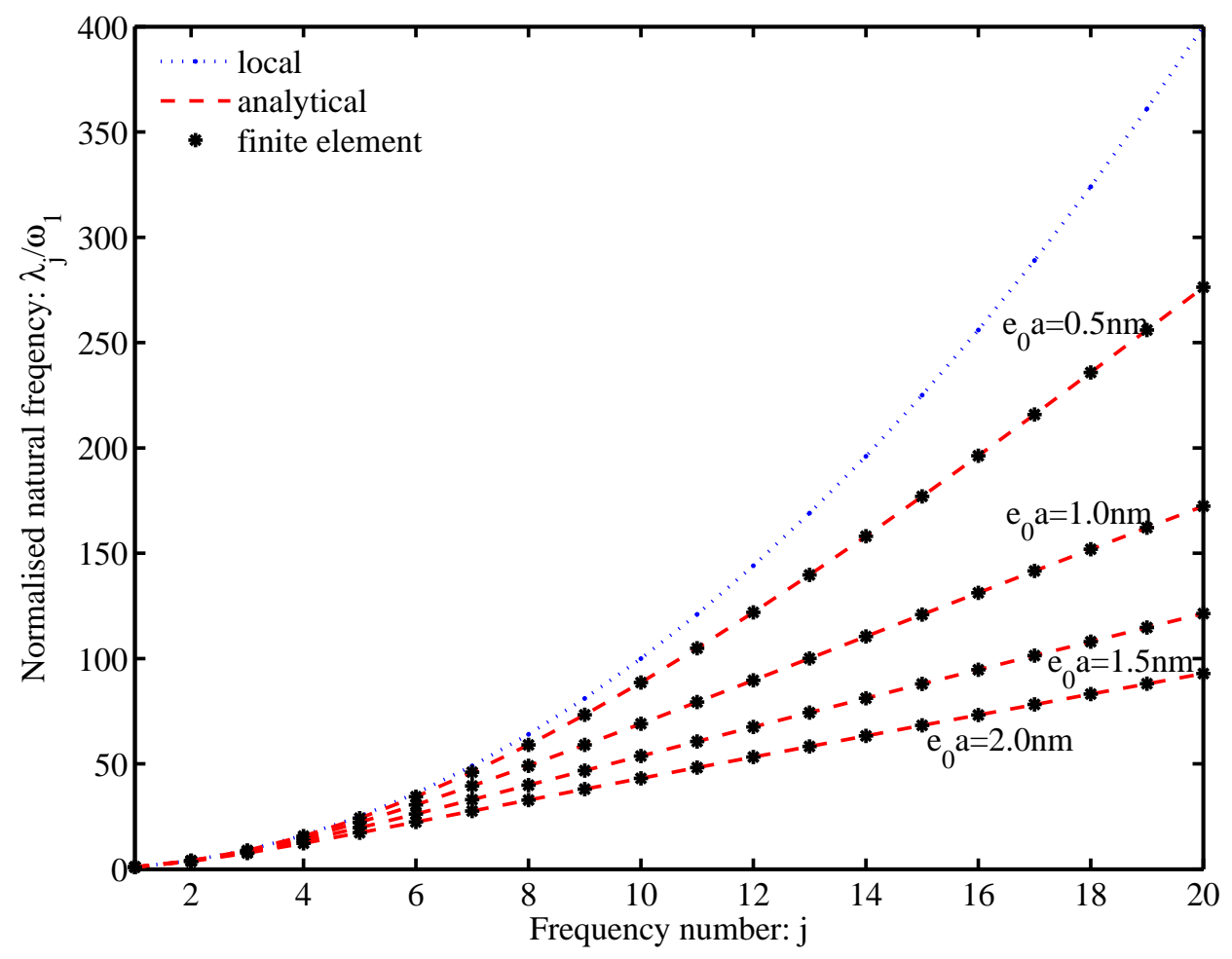

Fig. 9: The variation of the first 20 undamped natural frequencies for the bending vibration of DWCNT with pinned-pinned boundary condition and foundation stiffness $k=0$. Four representative values of $e_{0} a$ (in $\mathrm{nm}$ ) are considered.

boundary conditions we obtain the matrix equation

$$
\left[\begin{array}{cc}
D_{22}(\omega) & D_{24}(\omega) \\
D_{42}(\omega) & D_{44}(\omega)
\end{array}\right]\left\{\begin{array}{c}
\left.\theta_{1} \omega\right) \\
\theta_{2}(\omega)
\end{array}\right\}=\left\{\begin{array}{c}
0 \\
M
\end{array}\right\}
$$

The above equation arises due to the fact that degrees of freedom 1 and 3 (refer to Fig. 2) are zero. Using the analytical expressions of the matrix coefficients we have

$$
\frac{E I\left(\alpha^{2}+\beta^{2}\right)}{\Delta}\left[\begin{array}{cc}
-C \beta s+\alpha c S & -\alpha S+\beta s \\
-\alpha S+\beta s & -C \beta s+\alpha c S
\end{array}\right]\left\{\begin{array}{c}
\left.\theta_{1} \omega\right) \\
\theta_{2}(\omega)
\end{array}\right\}=\left\{\begin{array}{c}
0 \\
M
\end{array}\right\}
$$

Solving this equation, the damped dynamic response of the rotation at the right end (removing the subscript for simplicity) can be obtained in closed-form as

$$
\theta(\omega)=\frac{(\alpha s(C-1)(C+1) c+S(C) \beta(c-1)(c+1)) M}{E I\left(\alpha^{2}+\beta^{2}\right)\left(C^{2}-1\right)\left(c^{2}-1\right)}
$$

Taking the limiting case when the excitation frequency is zero (static case), we have

$$
\lim _{\alpha, \beta \rightarrow 0} \theta(\omega)=\theta_{\text {st }}=\frac{M L}{3 E I}
$$

This matches exactly with the well known classical expression [60].

In Fig. 10 the amplitude of the dynamic rotation $\theta(\omega)$ at the right-end is shown for the four representative values of the nonlocal parameter. We consider the mass and stiffness proportional damping such that the damping factors $\zeta_{2}=0.05$ and $\zeta_{1}=10^{-4}$. In the 


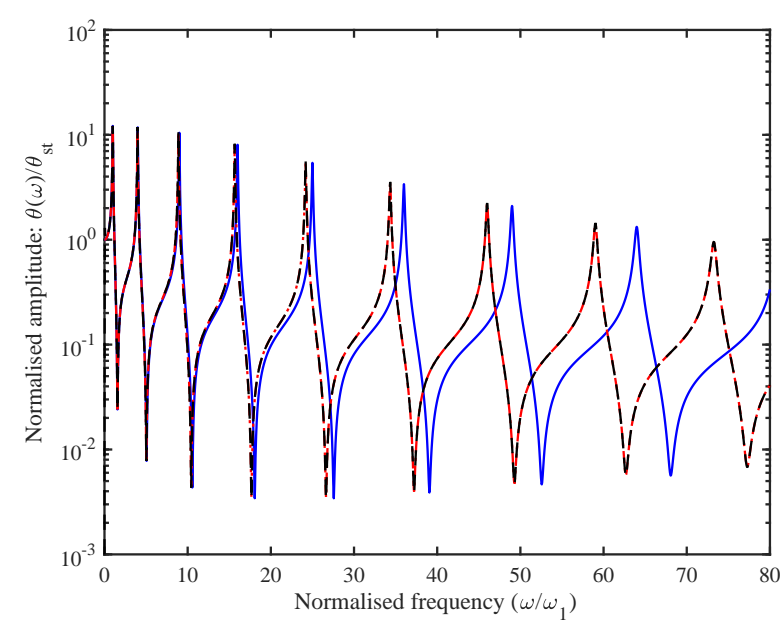

(a) $e_{0} a=0.5 \mathrm{~nm}$

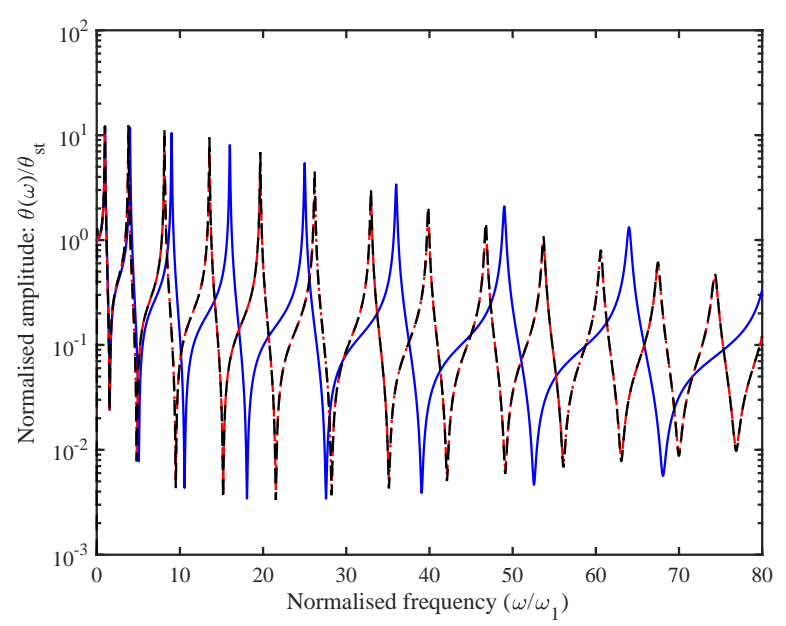

(c) $e_{0} a=1.5 \mathrm{~nm}$

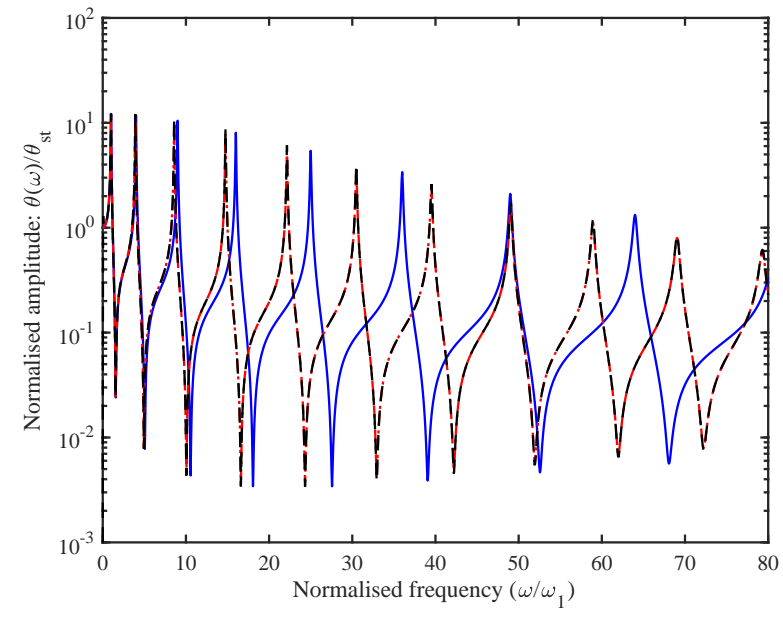

(b) $e_{0} a=1.0 \mathrm{~nm}$

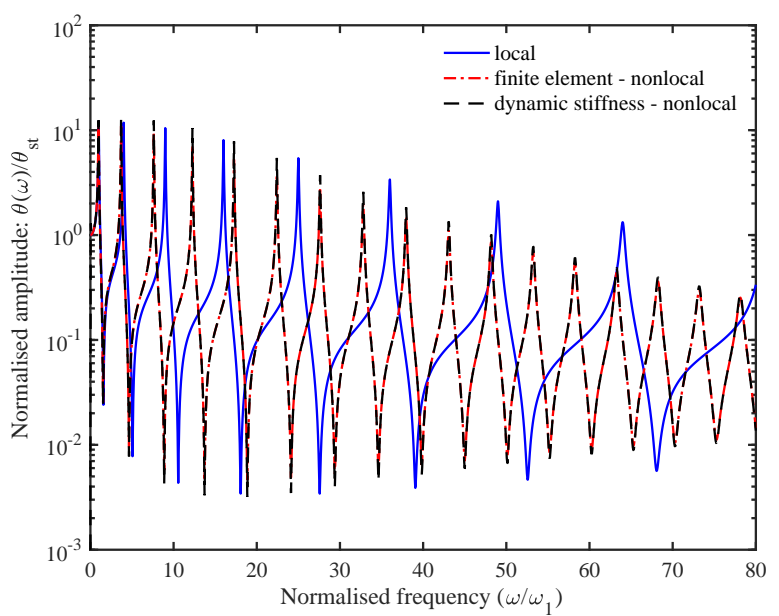

(d) $e_{0} a=2.0 \mathrm{~nm}$

Fig. 10: Amplitude of the normalised frequency response of the DWCNT $\theta(\omega)$ at the right-end for different values of $e_{0} a$ for pinned-pinned boundary condition. Exact finite element results are compared with the approximate analysis based on local eigensolutions. The foundation stiffness $k=0$ and the stiffness and mass proportional damping factors are $\zeta_{1}=10^{-4}$ and $\zeta_{2}=0.05$ respectively.

$\mathrm{x}$-axis, excitation frequency normalised with respect to the first local frequency given in Eq. (76) is considered. The frequency response is normalised by the static response $\theta_{\text {st }}$ in Eq. (82). The frequency response function of the underlying local model is also plotted to show the difference between the local and nonlocal response. For the nonlocal system, the frequency response is obtained by the direct finite element method and the dynamic stiffness method. For the finite element analysis we used 100 elements. This in turn, results in global mass and stiffness matrices of dimension $200 \times 200$. While for the dynamic stiffness method, only the inversion of a $2 \times 2$ matrix is necessary. The results demonstrates the computational efficiency and accuracy of the proposed dynamics stiffness method over the conventional finite element method.

Once the accuracy of the proposed dynamic stiffness method has been established, the interest now is to obtain dynamic response of the DWCNT in the cantilever configuration (figure not shown). A harmonic force is applied at the right end of the beam and we are 
interested in the dynamic response at the right-end. The left-end of the beam is fixed. Considering the dynamic equilibrium in Eq. (45) and applying the boundary conditions we obtain the matrix equation

$$
\left[\begin{array}{ll}
D_{33}(\omega) & D_{34}(\omega) \\
D_{43}(\omega) & D_{44}(\omega)
\end{array}\right]\left\{\begin{array}{c}
\left.v_{2} \omega\right) \\
\theta_{2}(\omega)
\end{array}\right\}=\left\{\begin{array}{l}
P \\
0
\end{array}\right\}
$$

The above equation arises due to the fact that degrees of freedom 1 and 2 (refer to Fig. 2) are zero. Solving this equation, the damped dynamic response of the displacement at the right end (removing the subscript for simplicity) can be obtained in closed-form as

$$
\begin{gathered}
v(\omega)=P\left(\alpha^{2}+\beta^{2}\right)\left(C^{2} \alpha^{2} \beta^{3} c s+C^{2} \beta^{5} c s-C S \alpha^{5} c^{2}-C S \alpha^{3} \beta^{2} c^{2}+C S \alpha^{3} \beta^{2}\right. \\
\left.-C S \alpha \beta^{4}+2 C \alpha^{2} \beta^{3} s-2 S \alpha^{3} \beta^{2} c+\alpha^{4} \beta c s-\alpha^{2} \beta^{3} c s\right) / \\
E I \alpha\left(C^{2} \alpha^{8} c^{2}+C^{2} \alpha^{6} \beta^{2} c^{2}+C^{2} \alpha^{2} \beta^{6} c^{2}+C^{2} \beta^{8} c^{2}-C^{2} \alpha^{6} \beta^{2}+2 C^{2} \alpha^{4} \beta^{4}-C^{2} \alpha^{2} \beta^{6}\right. \\
\left.+4 C \alpha^{6} \beta^{2} c+4 C \alpha^{2} \beta^{6} c-\alpha^{6} \beta^{2} c^{2}+2 \alpha^{4} \beta^{4} c^{2}-\alpha^{2} \beta^{6} c^{2}+\alpha^{6} \beta^{2}+2 \alpha^{4} \beta^{4}+\alpha^{2} \beta^{6}\right) \beta
\end{gathered}
$$

Taking the limiting case when the excitation frequency is zero (the static case), we have

$$
\lim _{\alpha, \beta \rightarrow 0} v(\omega)=v_{\mathrm{st}}=\frac{P L^{3}}{3 E I}
$$

This matches exactly with the well known classical expression [60].

In Fig. 11 the amplitude of the dynamic displacement $v(\omega)$ at the right-end is shown for the four representative values of the foundation stiffness $k=\omega_{s}^{2} m$. As the response quantity is a complex valued function, in Fig. 12 the corresponding phase of the response is shown. We consider the mass and stiffness proportional damping such that the damping factors $\zeta_{2}=0.05$ and $\zeta_{1}=10^{-4}$. In the x-axis, excitation frequency normalised with respect to the first local frequency as before. The frequency response is normalised by the static response $v_{\mathrm{st}}=\frac{P L^{3}}{3 E I}$ as given in Eq. (85). The increasing value of the nonlocal parameter results in a reduced natural frequency as observed before. However, interestingly we observe an increase in the damping effect with the increasing value of the nonlocal parameter even when the same damping factors are used in all the plots. This can be observed from a diminishing response amplitude for higher values of the nonlocal parameter. This damping effect is particularly prominent at higher frequencies and it is independent of the foundation stiffness. For higher values of the foundation stiffness, we observe that the first few resonance frequencies shifts to the left. This is expected as the system becomes stiffer. The impact of the foundation stiffness is less at the high-frequency ranges. The phase response in Fig. 12 corresponds to the characteristics of the amplitude response. A significant phase change is observed around the resonance peaks as expected. As the amplitude response becomes flatter for higher values of the nonlocal parameter, a diminishing change in the phase is observed. The analysis conducted here demonstrates that the dynamic stiffness approach developed here can be used to investigate the dynamics of damped nonlocal nano-beams without conducting a modal analysis. As the dynamic stiffness elements are exact, they are valid for all frequency ranges.

\section{Conclusions}

A novel dynamic finite element approach for bending vibration of damped nonlocal beams has been proposed. Strain rate dependent viscous damping and velocity-dependent 


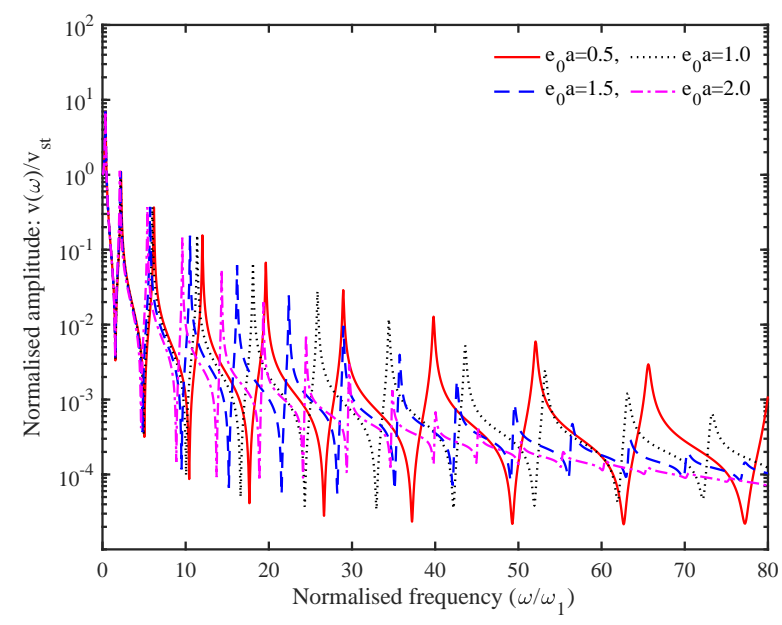

(a) $\omega_{s}=0$ (no elastic foundation)

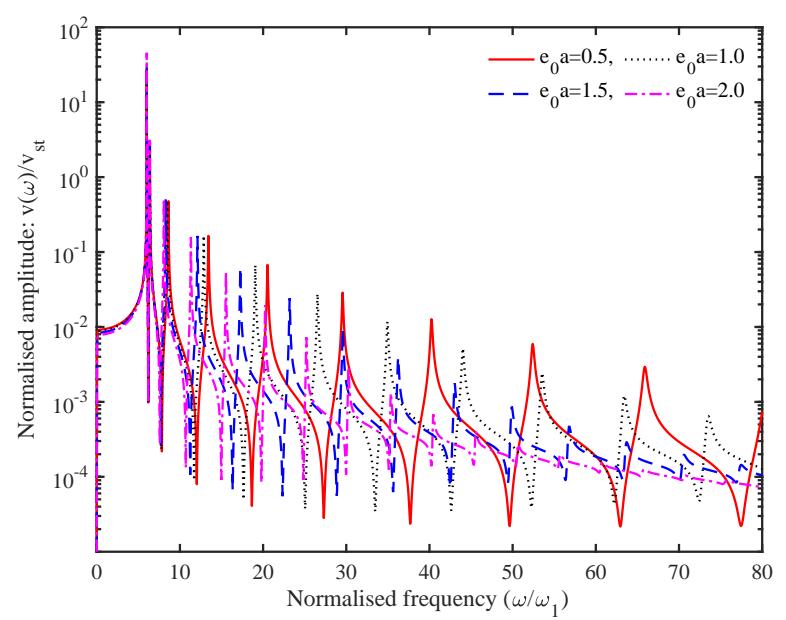

(c) $\omega_{s}=6.0$

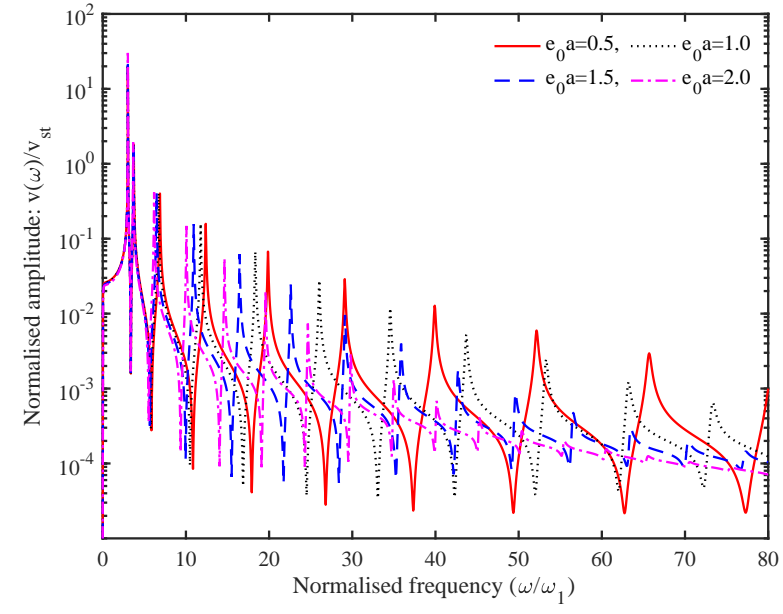

(b) $\omega_{s}=3.0$

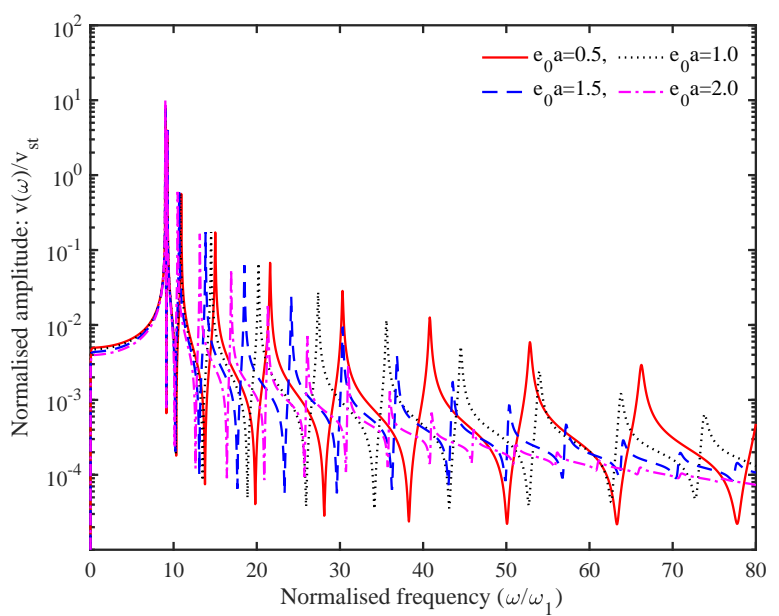

(d) $\omega_{s}=9.0$

Fig. 11: Amplitude of the normalised frequency response of the DWCNT $v(\omega)$ at the right-end for four different values of foundation stiffness $k=\omega_{s}^{2} m$. A cantilever boundary condition with a harmonic forcing at the right-end is considered. In each plot, we have shown four values of the nonlocal parameter. The stiffness and mass proportional damping factors are $\zeta_{1}=10^{-4}$ and $\zeta_{2}=0.05$ respectively.

viscous damping were employed. It was considered that the beam is resting on an elastic foundation. Damped and undamped dynamics were discussed. The stiffness and mass matrices of the nonlocal beam were obtained using the conventional finite element method. In the special case when the nonlocal parameter becomes zero, the expression of the mass and stiffness matrices reduce to the classical case. Frequency-dependent complex-valued shape functions were used to obtain the dynamic stiffness matrix in closed-form. It was shown that the $4 \times 4$ complex symmetric dynamic stiffness matrix is uniquely characterised by six transcendental functions. It was proved that, when the nonlocal parameter is zero (classical beam), the foundation stiffness is zero (no elastic foundation), frequency is zero (the static case), or the damping is zero (undamped case), the general dynamic stiffness matrix reduces to the well established special cases available in the literature. The dynamic stiffness matrix derived here, therefore, embodies the most general case within the scope of liner Euler-Bernoulli beam theory. 


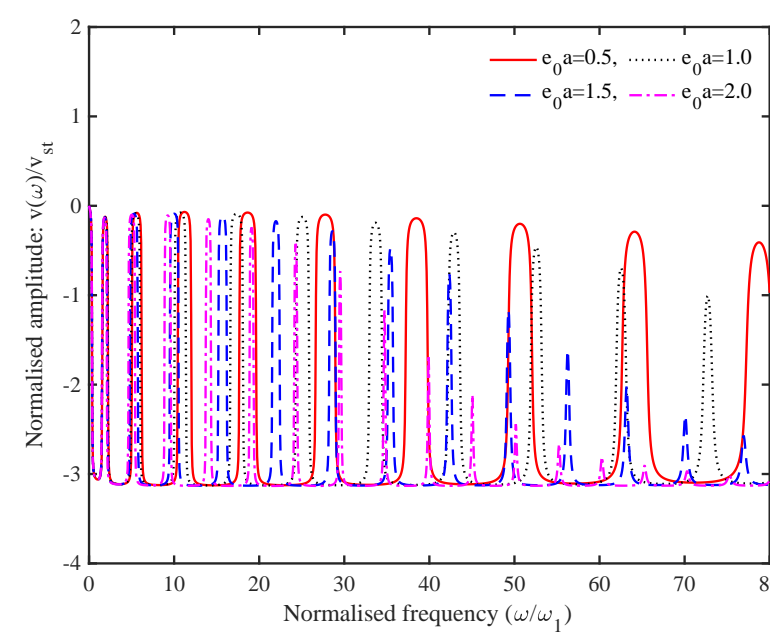

(a) $\omega_{s}=0$ (no elastic foundation)

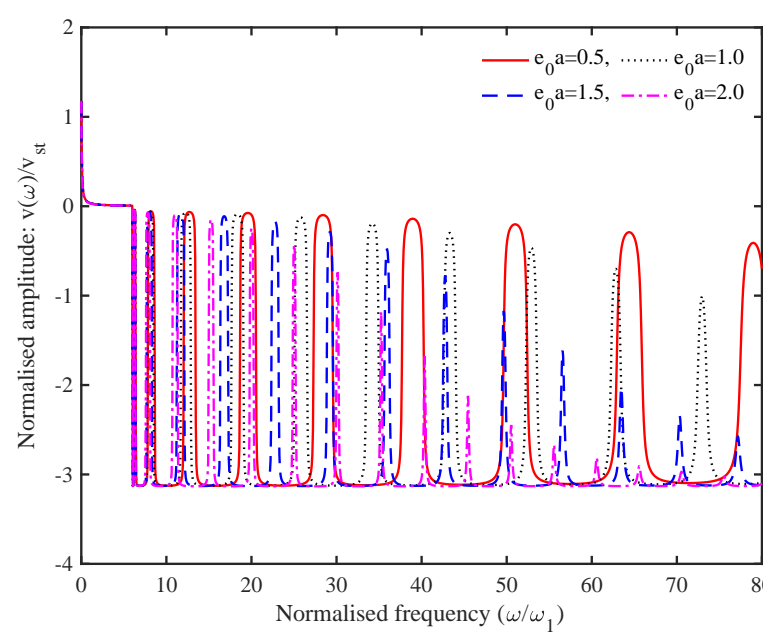

(c) $\omega_{s}=6.0$

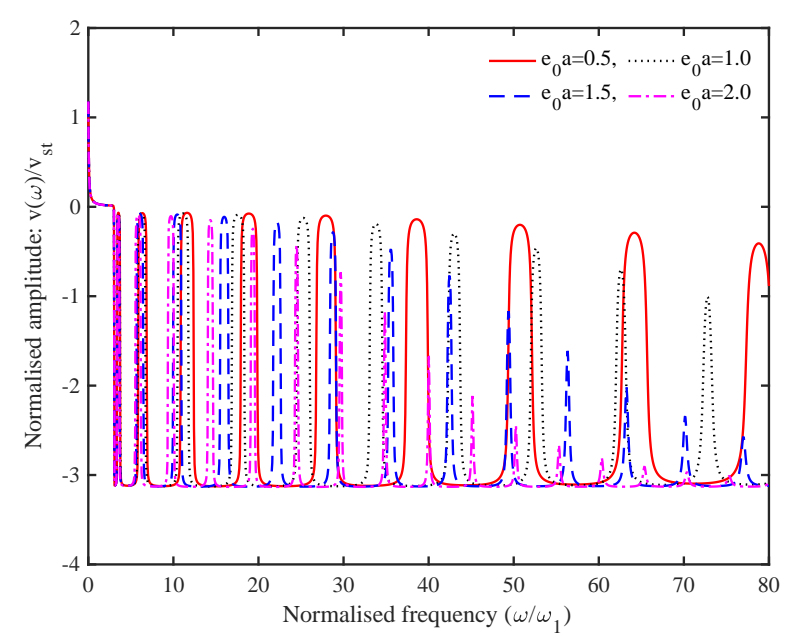

(b) $\omega_{s}=3.0$

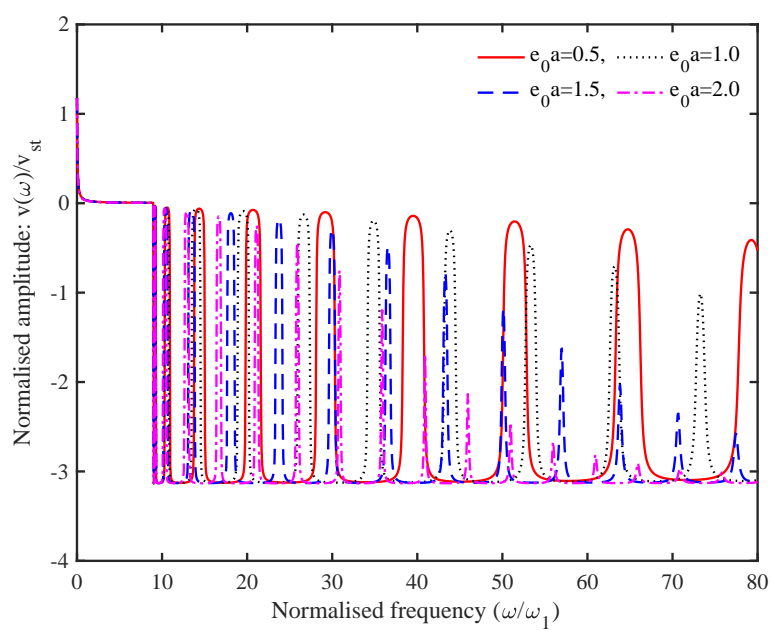

(d) $\omega_{s}=9.0$

Fig. 12: Phase of the normalised frequency response of the DWCNT $v(\omega)$ at the right-end for four different values of foundation stiffness $k=\omega_{s}^{2} m$. A cantilever boundary condition with a harmonic forcing at the right-end is considered. In each plot, we have shown four values of the nonlocal parameter. The stiffness and mass proportional damping factors are $\zeta_{1}=10^{-4}$ and $\zeta_{2}=0.05$ respectively.

The proposed method was numerically applied to the bending vibration of an armchair $(5,5),(8,8)$ double-walled carbon nanotube. The evolution of the elements of the dynamic stiffness matrix in the frequency domain has been shown for selected parameter values. Although the six dynamic stiffness coefficients are different, they show peaks at the same frequency values. Increasing values of the nonlocal parameter tend to make the stiffness coefficients softer while increasing values of the foundation stiffness tend to make the stiffness coefficients stiffer. However, the impact of the foundation stiffness is only prominent in the first few modes of vibration. Among the two types of damping considered in the paper, the strain rate dependent viscous damping has the most significant influence on the response behaviour in the high-frequency region. The dynamic response in the frequency domain was obtained by inverting the dynamic stiffness matrix with appropriate end conditions. Explicit closed-form expressions of the dynamic response for pinned-pinned and cantilever boundary conditions were obtained exactly in 
terms of transcendental functions. It was shown that in the special case of the static response, these complex expressions reduce to known simple classical formulas. The natural frequencies and the dynamic response obtained using the conventional finite element approach were compared with the results obtained using the dynamic finite method for the pinned-pinned boundary condition. Good agreement between conventional finite element with 100 elements and proposed dynamic finite element with only 1 element was found. This demonstrated the accuracy and computational efficiency of the proposed dynamic stiffness method.

\section{Acknowledgements}

SA and DK gratefully acknowledges the financial support from the European Commission under the Marie Skodowska Curie Actions (grant number 799201- METACTIVE). XL acknowledges the financial supports from the National Natural Science Foundation of China (Grant No. 11802345) and State Key Laboratory of High Performance Complex Manufacturing (Grant No. ZZYJKT2019-07). SA and XL also acknowledges the financial support from the High-end foreign expert introduction project through Grant no G20190018004.

\section{References}

[1] M. Eltaher, M. Khater, S. A. Emam, A review on nonlocal elastic models for bending, buckling, vibrations, and wave propagation of nanoscale beams, Applied Mathematical Modelling 40 (5-6) (2016) 4109-4128.

[2] A. Farajpour, M. H. Ghayesh, H. Farokhi, A review on the mechanics of nanostructures, International Journal of Engineering Science 133 (2018) 231-263.

[3] L. Schmidt-Mende, J. L. MacManus-Driscoll, Zno-nanostructures, defects, and devices, Materials today 10 (5) (2007) 40-48.

[4] R. H. Baughman, A. A. Zakhidov, W. A. De Heer, Carbon nanotubes-the route toward applications, science 297 (5582) (2002) 787-792.

[5] L. M. S. Ansaloni, E. M. B. de Sousa, Boron nitride nanostructured: Synthesis, characterization and potential use in cosmetics, Materials sciences and applications 4 (01) (2013) 22.

[6] A. Fulati, S. Usman Ali, M. Riaz, G. Amin, O. Nur, M. Willander, Miniaturized PH sensors based on zinc oxide nanotubes/nanorods, Sensors 9 (11) (2009) 8911-8923.

[7] I. Bargatin, E. Myers, J. Aldridge, C. Marcoux, P. Brianceau, L. Duraffourg, E. Colinet, S. Hentz, P. Andreucci, M. Roukes, Large-scale integration of nanoelectromechanical systems for gas sensing applications, Nano letters 12 (3) (2012) 1269-1274.

[8] J. G. Park, C. Zhang, R. Liang, B. Wang, Nano-machining of highly oriented pyrolytic graphite using conductive atomic force microscope tips and carbon nanotubes, Nanotechnology 18 (40) (2007) 405306.

[9] H.-J. Kim, A. Emge, R. Winter, P. Keightley, W.-K. Kim, M. Falk, D. Rigney, Nanostructures generated by explosively driven friction: Experiments and molecular dynamics simulations, Acta Materialia 57 (17) (2009) 5270-5282.

[10] A. C. Eringen, On differential equations of nonlocal elasticity and solutions of screw dislocation and surface waves, Journal of applied physics 54 (9) (1983) 4703-4710.

[11] A. C. Eringen, Linear theory of nonlocal elasticity and dispersion of plane waves, International Journal of Engineering Science 10 (5) (1972) 425-435.

[12] J. Peddieson, G. R. Buchanan, R. P. McNitt, Application of nonlocal continuum models to nanotechnology, International Journal of Engineering Science 41 (3-5) (2003) 305-312.

[13] T. Murmu, S. Adhikari, Nonlocal transverse vibration of double-nanobeam-systems, Journal of Applied Physics 108 (8) (2010) 083514. 
[14] T. Murmu, M. McCarthy, S. Adhikari, Vibration response of double-walled carbon nanotubes subjected to an externally applied longitudinal magnetic field: a nonlocal elasticity approach, Journal of Sound and Vibration 331 (23) (2012) 5069-5086.

[15] J. Reddy, Nonlocal theories for bending, buckling and vibration of beams, International Journal of Engineering Science 45 (2-8) (2007) 288-307.

[16] J. Reddy, S. Pang, Nonlocal continuum theories of beams for the analysis of carbon nanotubes, Journal of Applied Physics 103 (2) (2008) 023511.

[17] M. Eltaher, A. E. Alshorbagy, F. Mahmoud, Vibration analysis of Euler-Bernoulli nanobeams by using finite element method, Applied Mathematical Modelling 37 (7) (2013) 4787-4797.

[18] J. Phadikar, S. Pradhan, Variational formulation and finite element analysis for nonlocal elastic nanobeams and nanoplates, Computational materials science 49 (3) (2010) 492-499.

[19] M. Tuna, M. Kirca, Bending, buckling and free vibration analysis of euler-bernoulli nanobeams using eringens nonlocal integral model via finite element method, Composite Structures 179 (2017) 269-284.

[20] S. Pradhan, Nonlocal finite element analysis and small scale effects of CNTs with timoshenko beam theory, Finite Elements in Analysis and Design 50 (2012) 8-20.

[21] S. Adhikari, T. Murmu, M. McCarthy, Dynamic finite element analysis of axially vibrating nonlocal rods, Finite Elements in Analysis and Design 63 (2013) 42-50.

[22] S. Adhikari, T. Murmu, M. McCarthy, Frequency domain analysis of nonlocal rods embedded in an elastic medium, Physica E: Low-dimensional Systems and Nanostructures 59 (2014) 33-40.

[23] S. Adhikari, D. Gilchrist, T. Murmu, M. McCarthy, Nonlocal normal modes in nanoscale dynamical systems, Mechanical Systems and Signal Processing 60 (2015) 583-603.

[24] J. F. Doyle, Wave Propagation in Structures, Springer Verlag, New York, 1989.

[25] M. Paz, Structural Dynamics: Theory and Computation, 2nd Edition, Van Nostrand, Reinhold, 1980.

[26] J. R. Banerjee, Coupled bending torsional dynamic stiffness matrix for beam elements, International Journal for Numerical Methods in Engineering 28 (6) (1989) 1283-1298.

[27] C. S. Manohar, S. Adhikari, Dynamic stiffness of randomly parametered beams, Probabilistic Engineering Mechanics 13 (1) (1998) 39-51.

[28] X. Liu, J. Banerjee, An exact spectral-dynamic stiffness method for free flexural vibration analysis of orthotropic composite plate assemblies - Part I: Theory, Composite Structures 132 (2015) 1274-1287.

[29] X. Liu, J. Banerjee, A spectral dynamic stiffness method for free vibration analysis of plane elastodynamic problems, Mechanical Systems and Signal Processing 87 (2017) 136-160.

[30] J. R. Banerjee, Dynamic stiffness formulation for structural elements: A general approach, Computer and Structures 63 (1) (1997) 101-103.

[31] S. Adhikari, C. S. Manohar, Transient dynamics of stochastically parametered beams, ASCE Journal of Engineering Mechanics 126 (11) (2000) 1131-1140.

[32] S. Gopalakrishnan, A. Chakraborty, D. R. Mahapatra, Spectral Finite Element Method, Springer Verlag, New York, 2007.

[33] S. M. Hashemi, M. J. Richard, G. Dhatt, A new Dynamic Finite Element (DFE) formulation for lateral free vibrations of Euler-Bernoulli spinning beams using trigonometric shape functions, Journal of Sound and Vibration 220 (4) (1999) 601-624.

[34] S. M. Hashemi, M. J. Richard, Free vibrational analysis of axially loaded bending-torsion coupled beams: a dynamic finite element, Computer and Structures 77 (6) (2000) 711-724.

[35] Y. Zhang, C. Wang, N. Challamel, Bending, buckling, and vibration of micro/nanobeams by hybrid nonlocal beam model, Journal of Engineering Mechanics 136 (5) (2010) 562-574.

[36] J. N. Reddy, Nonlocal theories for bending, buckling and vibration of beams, International Journal of Engineering Science 45 (2-8) (2007) 288-307.

[37] P. Karaoglu, M. Aydogdu, On the forced vibration of carbon nanotubes via a non-local eulerbernoulli beam model, Proceedings of the Institution of Mechanical Engineers Part C-Journal of Mechanical Engineering Science 224 (C2) (2010) 497-503.

[38] Z. Zhang, C. Wang, N. Challamel, Eringens length scale coefficient for buckling of nonlocal rectangular plates from microstructured beam-grid model, International Journal of Solids and Structures $51(25-26)(2014)$ 4307-4315.

[39] C. Wang, Z. Zhang, N. Challamel, W. Duan, Calibration of Eringen's small length scale coefficient for initially stressed vibrating nonlocal Euler beams based on microstructured beam model, Journal 
of Physics D: Applied Physics 46 (34) (2013) 345501.

[40] M. I. Friswell, S. Adhikari, Y. Lei, Nonlocal finite element analysis of damped beams, International Journal of Solids and Structures 44 (22-23) (2007) 7564-7576.

[41] C. Koutsoumaris, K. Eptaimeros, G. Tsamasphyros, A different approach to eringen's nonlocal integral stress model with applications for beams, International Journal of Solids and Structures 112 (2017) $222-238$.

[42] L. Meirovitch, Principles and Techniques of Vibrations, Prentice-Hall International, Inc., New Jersey, 1997.

[43] N. Challamel, J. Reddy, C. Wang, Eringen's stress gradient model for bending of nonlocal beams, Journal of Engineering Mechanics 142 (12) (2016) 04016095.

[44] J. Fernández-Sáez, R. Zaera, J. Loya, J. Reddy, Bending of Euler-Bernoulli beams using Eringens integral formulation: a paradox resolved, International Journal of Engineering Science 99 (2016) $107-116$.

[45] N. Challamel, C. Wang, The small length scale effect for a non-local cantilever beam: a paradox solved, Nanotechnology 19 (34) (2008) 345703.

[46] P. Khodabakhshi, J. Reddy, A unified integro-differential nonlocal model, International Journal of Engineering Science 95 (2015) 60-75.

[47] G. Romano, R. Barretta, Stress-driven versus strain-driven nonlocal integral model for elastic nanobeams, Composites Part B: Engineering 114 (2017) 184-188.

[48] G. Romano, R. Barretta, Nonlocal elasticity in nanobeams: the stress-driven integral model, International Journal of Engineering Science 115 (2017) 14-27.

[49] N. Challamel, Z. Zhang, C. Wang, J. Reddy, Q. Wang, T. Michelitsch, B. Collet, On nonconservativeness of eringens nonlocal elasticity in beam mechanics: correction from a discrete-based approach, Archive of Applied Mechanics 84 (9-11) (2014) 1275-1292.

[50] C. Wang, H. Zhang, N. Challamel, W. Duan, On boundary conditions for buckling and vibration of nonlocal beams, European Journal of Mechanics - A/Solids 61 (2017) 73 - 81.

[51] J. K. Phadikar, S. C. Pradhan, Variational formulation and finite element analysis for nonlocal elastic nanobeams and nanoplates, Computational Materials Science 49 (3) (2010) 492-499.

[52] M. Petyt, Introduction to Finite Element Vibration Analysis, Cambridge University Press, Cambridge, UK, 1998.

[53] D. Dawe, Matrix and Finite Element Displacement Analysis of Structures, Oxford University Press, Oxford, UK, 1984.

[54] S. Adhikari, Doubly spectral stochastic finite element method (DSSFEM) for structural dynamics, ASCE Journal of Aerospace Engineering 24 (3) (2011) 264-276.

[55] A. Y. T. Leung, Dynamic Stiffness and Substructures, Springer-Verlag, London, UK, 1993.

[56] W. Wittrick, F. Williams, A general algorithm for computing natural conditions conditions of elastic structures, The Quarterly Journal of Mechanics and Applied Mathematics 24 (3) (1971) 263-284.

[57] X. Liu, J. Banerjee, Free vibration analysis for plates with arbitrary boundary conditions using a novel spectral-dynamic stiffness method, Computers \& Structures 164 (2016) 108-126.

[58] T. Murmu, S. Adhikari, M. McCarthy, Vibration response of double-walled carbon nanotubes subjected to an externally applied longitudinal magnetic field: A nonlocal elasticity approach, Journal of Sound and Vibration 331 (23) (2012) 5069-5086.

[59] M. Aydogdu, Axial vibration of the nanorods with the nonlocal continuum rod model, Physica E 41 (5) (2009) 861-864.

[60] Young W C, Budynas R G, Roark's Formulas for Stresses and Strains, 7th Edition, McGraw-Hill, Inc, London, 2002. 